\title{
Removal of the resolvent-like energy dependence from interactions and invariant subspaces of a total Hamiltonian
}

\author{
A.K.Motovilov \\ Laboratory of Theoretical Physics, JINR, 141980 Dubna, Moscow region, Russia \\ E-address: motovilv@thsun1.jinr.dubna.su
}

The spectral problem $(A+V(z)) \psi=z \psi$ is considered where the main Hamiltonian $A$ is a self-adjoint operator of sufficiently arbitrary nature. The perturbation $V(z)=-B\left(A^{\prime}-z\right)^{-1} B^{*}$ depends on the energy $z$ as resolvent of another self-adjoint operator $A^{\prime}$. The latter is usually interpreted as Hamiltonian describing an internal structure of physical system. The operator $B$ is assumed to have a finite Hilbert-Schmidt norm. The conditions are formulated when one can replace the perturbation $V(z)$ with an energy-independent "potential" $W$ such that the Hamiltonian $H=A+W$ has the same spectrum (more exactly a part of spectrum) and the same eigenfunctions as the initial spectral problem. The Hamiltonian $H$ is constructed as a solution of the nonlinear operator equation $H=A+V(H)$. It is established that this equation is closely connected with the problem of searching for invariant subspaces of the Hamiltonian $\mathbf{H}=\left[\begin{array}{ll}A & B \\ B^{*} & A^{\prime}\end{array}\right]$. The orthogonality and expansion theorems are proved for eigenfunction systems of the Hamiltonian $H=A+W$. Scattering theory is developed for this Hamiltonian in the case where the operator $A$ has continuous spectrum.

AMS subject classification (1980) 47B99, 81G05.

Published in J. Math. Phys., 1995, vol. 36 (12), pp. 6647-6664. LANL E-print funct-an/9606002.

\section{INTRODUCTION}

Perturbations, depending on the spectral parameter (usually energy of system) arise in a lot of physical problems (see papers [1]- [16 and Refs. therein). In particular, such are the interaction potentials between clusters formed by quantum particles [1]- [6].

The perturbations of this type appear typically [1]- [4], [11]- [16] as a result of dividing the Hilbert space $\mathcal{H}$ of physical system in two subspaces, $\mathcal{H}=\mathcal{H}_{1} \oplus \mathcal{H}_{2}$. The first one, say $\mathcal{H}_{1}$, is interpreted as a space of "external" (for example, hadronic) degrees of freedom. The second one, $\mathcal{H}_{2}$, is associated with an "internal" (for example, quark) structure of the system. The Hamiltonian $\mathbf{H}$ of the system looks as a matrix,

$$
\mathbf{H}=\left[\begin{array}{cc}
A_{1} & B_{12} \\
B_{21} & A_{2}
\end{array}\right]
$$


with $A_{1}, A_{2}$, the channel Hamiltonians (self-adjoint operators) and $B_{12}, B_{21}=B_{12}^{*}$, the coupling operators. Reducing the spectral problem $\mathbf{H} U=z U, U=\left\{u_{1}, u_{2}\right\}$ to the channel $\alpha$ only one gets the spectral problem

$$
\left[A_{\alpha}+V_{\alpha}(z)\right] u_{\alpha}=z u_{\alpha}, \quad \alpha=1,2,
$$

where the perturbation

$$
V_{\alpha}(z)=-B_{\alpha \beta}\left(A_{\beta}-z\right)^{-1} B_{\beta \alpha}, \quad \beta \neq \alpha,
$$

depends on the spectral parameter $z$ as the resolvent $\left(A_{\beta}-z\right)^{-1}$ of the Hamiltonian $A_{\beta}$. In more complicated cases $V_{\alpha}(z)$ can include also linear terms in respect with $z$. Other types of dependency of the potentials $V_{\alpha}(z)$ on the spectral parameter z give, in a general way, the spectral problems (2) with a complex spectrum.

The present paper is a continuation of the author's works [17]- [19] devoted to a study of the possibility to "remove" the energy dependence from perturbations of the type (3). Namely, in [17] - [19] we construct such new potential $W_{\alpha}$ that spectrum of the Hamiltonian $H_{\alpha}=A_{\alpha}+W_{\alpha}$ is a part of the spectrum of the problem (2). At the same time, the respective eigenvectors of $H_{\alpha}$ become also those for (2). Hamiltonians $H_{\alpha}$ are found as solutions of the non-linear operator equations

$$
H_{\alpha}=A_{\alpha}+V_{\alpha}\left(H_{\alpha}\right)
$$

first appeared in the paper [9] by M.A.Braun in connection with consideration of the quasipotential equation. The operator-value function $V_{\alpha}(Y)$ of the operator variable $Y$, $Y: \mathcal{H}_{\alpha} \rightarrow \mathcal{H}_{\alpha}$, is defined by us in such a way (see Sec. III) that eigenvectors $\psi$ of $Y$, $Y \psi=z \psi$, become automatically those for $V_{\alpha}(Y)$ and $V_{\alpha}(Y) \psi=V_{\alpha}(z) \psi$.

Note that the problem of removal of the dependence on a spectral parameter from perturbation is interesting not only in itself. In particular, there is a rather conceptual question (see for instance Refs. [7], [10] and [17]) concerning a use of the two-body energy-dependent potentials in few-body nonrelativistic scattering problems. Since the energies of pair subsystems are not fixed in the $\mathrm{N}$-body $(\mathrm{N} \geq 3)$ problem, a direct embedding of such potentials into the few-body Hamiltonian is impossible. Thus, the replacements of the type (3) energydependent potentials with the respective new potentials $W_{\alpha}$ could be considered as a way to overcome this difficulty.

In Ref. [17], the problem of the removal of the energy dependence from the type (3) perturbations was considered in details when one of the operators $A_{\alpha}$ is the Schrödinger operator in $L_{2}\left(\mathbf{R}^{n}\right)$ and another one has a discrete spectrum only. The reports [18], 19] announce the results concerning the equations (4) and properties of their solutions $H_{\alpha}$ in a rather more general situation where the Hamiltonian $\mathbf{H}$ may be rewritten in terms of a twochannel variant of the Friedrichs model investigated by O.A.Ladyzhenskaya and L.D.Faddeev in Ref. [20] and L.D.Faddeev in Ref. [21]. In the paper [16] the method [17] -19] is applied for construction of an effective cluster Hamiltonian for atoms adsorbed by the metal surface.

In the present paper, we specify the assertions from [18], [19] and give proofs for them. Also, we pay attention to an important circumstance disclosing a nature of solutions of the basic equations (4). Thing is that the potentials $W_{\alpha}=V_{\alpha}\left(H_{\alpha}\right)$ may be presented in the form $W_{\alpha}=B_{\alpha \beta} Q_{\beta \alpha}$ where the operators $Q_{\beta \alpha}$ satisfy the stationary Riccati equations (24) 
(see Sec. III). Exactly the same equations arise in the method of construction of invariant subspaces developed by V.A.Malyshev and R.A.Minlos [22], 23] for a class of self-adjoint operators in statistical physics. It follows from the results of [22], [23] that operators $H_{\alpha}$, $\alpha=1,2$, determine in fact, parts of the two-channel Hamiltonian $\mathbf{H}$ acting in corresponding invariant subspaces (see Theorem 2 and comments to it).

Recently, the author came to know about the work [24] by V.M.Adamjan and H.Langer studying the operator-value functions written in our notation as $F_{\alpha}(z)=z-A_{\alpha} \pm B_{\alpha \beta}\left(A_{\beta}-\right.$ $z)^{-1} B_{\beta \alpha}$. In particular Adamjan and Langer show in this work that a subset of eigenvectors of $F_{\alpha}$ can be chosen to form a Riesz basis in $\mathcal{H}_{\alpha}$. There is a certain intersection of their results and ours from Refs. [17]- 19]. However the methods are different.

The paper is organized as follows.

In Sec. II we describe the Hamiltonian $\mathbf{H}$ as a two-channel variant of the Friedrichs model [20], 21]. We suppose that both operators $A_{\alpha}, \alpha=1,2$, may have continuous spectrum. When properties of objects connected with this spectrum (wave operators and scattering matrices) are considered in following sections, the coupling operators $B_{\alpha \beta}$ in (1) are assumed to be integral ones with kernels $B_{\alpha \beta}(\lambda, \mu)$, the Hölder functions in both variables $\lambda, \mu$.

In Sec. III the equations (41) are studied. As in Refs. [22], [23] we suppose that spectra $\sigma\left(A_{1}\right)$ and $\sigma\left(A_{2}\right)$ of the operators $A_{1}$ and $A_{2}$ are separated, $\operatorname{dist}\left\{\sigma\left(A_{1}\right), \sigma\left(A_{2}\right)\right\}>0$. Existence of solutions of Eqs. (国) is established only in the case where the Hilbert-Schmidt norms $\left\|B_{\alpha \beta}\right\|_{2}$ of the coupling operators $B_{\alpha \beta}$ satisfy the condition $\left\|B_{\alpha \beta}\right\|_{2}<\frac{1}{2} \operatorname{dist}\left\{\sigma\left(A_{1}\right), \sigma\left(A_{2}\right)\right\}$.

In Sec. IV the eigenfunctions systems of the operators $H_{\alpha}$ are studied and theorems of their orthogonality and completeness are proved. We show here in particular that spectrum of the Hamiltonian $\mathbf{H}$ is distributed between the solutions $H_{1}=A_{1}+B_{12} Q_{21}$ and $H_{2}=$ $A_{2}+B_{21} Q_{12}, Q_{21}=-Q_{12}^{*}$, of the basic equations (4) in such a way that $H_{1}$ and $H_{2}$ have not "common" eigenfunctions $U=\left\{u_{1}, u_{2}\right\}$ of $\mathbf{H}$ : simultaneously, the component $u_{1}$ can not be eigenfunction for $H_{1}$, and the component $u_{2}$, for $H_{2}$.

In Sec. $\mathrm{V}$ we introduce new inner products in the Hilbert spaces $\mathcal{H}_{\alpha}, \alpha=1,2$, making the Hamiltonians $H_{\alpha}$ self-adjoint.

In Sec. VI we give a non-stationary formulation of the scattering problem for a system described by the Hamiltonian $H_{\alpha}$ constructed in the case where $A_{\alpha}$ has continuous spectrum. We show that this formulation is correct and scattering operator is exactly the same as in initial spectral problem.

\section{INITIAL SPECTRAL PROBLEM AND TWO-CHANNEL HAMILTONIAN}

Let $A_{1}$ and $A_{2}$ be self-adjoint operators acting, respectively, in "external", $\mathcal{H}_{1}$, and "internal", $\mathcal{H}_{2}$, Hilbert spaces. We study the spectral problem (2) with perturbation $V_{\alpha}(z)$ given by (3). We suppose that $B_{\alpha \beta} \in \mathbf{B}\left(\mathcal{H}_{\alpha}, \mathcal{H}_{\beta}\right)$ where $\mathbf{B}\left(\mathcal{H}_{\alpha}, \mathcal{H}_{\beta}\right)$ is the Banach space of bounded linear operators acting from $\mathcal{H}_{\alpha}$ to $\mathcal{H}_{\beta}$.

Note that the method developed in the present paper works also in the case of more general perturbations $V_{\alpha}(z)=-\mathcal{R}_{\alpha}(z)$ containing linear terms,

$$
\mathcal{R}_{\alpha}(z)=N_{\alpha} z+B_{\alpha \beta}\left(A_{\beta}-N_{\beta} z-z\right)^{-1} B_{\beta \alpha}
$$


with $N_{\alpha}$, self-adjoint bounded operator in $\mathcal{H}_{\alpha}$ such that $N_{\alpha} \geq(\delta-1) I_{\alpha}$ where $\delta>0$ and $I_{\alpha}$ is the identity operator in $\mathcal{H}_{\alpha}$. Thing is that the equation (2) with $V_{\alpha}(z)=-\mathcal{R}_{\alpha}(z)$ can be easily rewritten in the form (2),(3). To do this, one has only to make the replacements $u_{\alpha} \rightarrow u_{\alpha}^{\prime}=\left(I_{\alpha}+N_{\alpha}\right)^{1 / 2} u_{\alpha}, \quad A_{\alpha} \rightarrow A_{\alpha}^{\prime}=\left(I_{\alpha}+N_{\alpha}\right)^{-1 / 2} A_{\alpha}\left(I_{\alpha}+N_{\alpha}\right)^{-1 / 2}$ and $B_{\alpha \beta} \rightarrow B_{\alpha \beta}^{\prime}=$ $\left(I_{\alpha}+N_{\alpha}\right)^{-1 / 2} B_{\alpha \beta}\left(I_{\beta}+N_{\beta}\right)^{-1 / 2}$. Therefore we shall consider further only the initial spectral problem (2), (3).

We shall assume that operators $A_{\alpha}, \alpha=1,2$, may have continuous spectra $\sigma_{\alpha}^{c}$. To deal with these spectra we accept below some presuppositions in respect with $A_{\alpha}$ restricting us to the case of a two-channel variant of the Friedrichs model [20], [21]. Note that these presuppositions are not necessary for a part of statements (Lemma 1, Theorems 11 3 and 5) which stay correct also in general case.

The presuppositions are following.

At first, we assume that Hamiltonian $H$ is defined in that representation where operators $A_{\alpha}, \alpha=1,2$, are diagonal. We suppose that continuous spectra $\sigma_{\alpha}^{c}$ of the operators $A_{\alpha}, \alpha=$ 1,2 , are absolutely continuous and consist of a finite number of finite (and may be one or two infinite) intervals $\left(a_{\alpha}^{(j)}, b_{\alpha}^{(j)}\right),-\infty \leq a_{\alpha}^{(j)}<b_{\alpha}^{(j)} \leq+\infty, j=1,2, \ldots, n_{\alpha}, n_{\alpha}<\infty$. At second, we suppose that discrete spectra $\sigma_{\alpha}^{d}$ of the operators $A_{\alpha}, \alpha=1,2$, do not intersect with $\sigma_{\alpha}^{c}, \sigma_{\alpha}^{d} \cap \sigma_{\alpha}^{c}=\emptyset$, and consist of a finite number of points with finite multiplicity. In this case the space $\mathcal{H}_{\alpha}$ may be present as the direct integral [27]

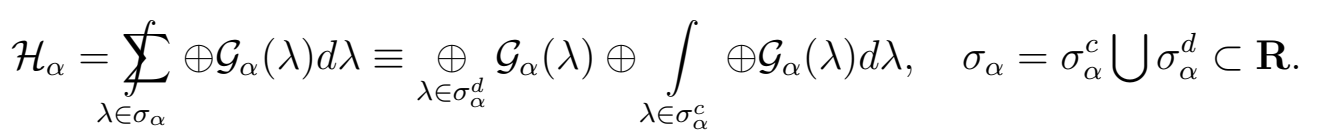

The space $\mathcal{H}_{\alpha}$ consists of the measurable functions $f_{\alpha}$ which are defined on $\sigma_{\alpha}$ and have the values $f_{\alpha}(\lambda)$ from corresponding Hilbert spaces $\mathcal{G}_{\alpha}(\lambda)$. By $\langle\cdot, \cdot\rangle$ we denote the inner product in $\mathcal{H}_{\alpha}$,

$$
\left\langle f_{\alpha}, g_{\alpha}\right\rangle=£_{\lambda \in \sigma_{\alpha}}\left(f_{\alpha}(\lambda), g_{\alpha}(\lambda)\right) \equiv \sum_{\lambda \in \sigma_{\alpha}^{d}}\left(f_{\alpha}(\lambda), g_{\alpha}(\lambda)\right)+\int_{\lambda \in \sigma_{\alpha}^{c}} d \lambda\left(f_{\alpha}(\lambda), g_{\alpha}(\lambda)\right),
$$

where $(\cdot, \cdot)$ stands for inner product in $\mathcal{G}_{\alpha}(\lambda)$. By $|\cdot|$ we denote norm of vectors and operators in $\mathcal{G}_{\alpha}(\lambda)$ and by $\|\cdot\|$, the norm in $\mathcal{H}_{\alpha}$. Operator $A_{\alpha}$ acts in $\mathcal{H}_{\alpha}$ as the independent variable multiplication operator,

$$
\left(A_{\alpha} f_{\alpha}\right)(\lambda)=\lambda \cdot f_{\alpha}(\lambda), \quad \alpha=1,2 .
$$

It's domain $\mathcal{D}\left(A_{\alpha}\right)$ consists of those functions $f_{\alpha} \in \mathcal{H}_{\alpha}$ which satisfy the condition $\sum_{\lambda \in \sigma_{\alpha}} \lambda^{2}\left|f_{\alpha}(\lambda)\right|^{2}<\infty$. For the sake of simplicity we assume that $\mathcal{G}_{\alpha}(\lambda)$ does not depend on $\lambda \in \sigma_{\alpha}^{c}$, i.e. $\mathcal{G}_{\alpha}(\lambda) \equiv \mathcal{G}_{\alpha}^{c}$ for each $\lambda \in \sigma_{\alpha}^{c}$. Hence, $\int_{\sigma_{\alpha}^{c}} \oplus \mathcal{G}_{\alpha}(\lambda) d \lambda=L_{2}\left(\sigma_{\alpha}^{c}, \mathcal{G}_{\alpha}^{c}\right) \equiv \mathcal{H}_{\alpha}^{c}$. By $E_{\alpha}(d \lambda)$ we denote a spectral measure [27] of the operator $A_{\alpha}, A_{\alpha}=\int_{\sigma_{\alpha}} \lambda E_{\alpha}(d \lambda)$. In the diagonal representation considered, the spectral projector $E_{\alpha}$ acts on $f \in \mathcal{H}_{\alpha}$ as

$$
\left(E_{\alpha}(\Delta) f\right)(\lambda)=\chi_{\Delta}(\lambda) f(\lambda)
$$

for any Borelian set $\Delta \subset \sigma_{\alpha}$. Here, $\chi_{\Delta}$ is a characteristic function of $\Delta, \chi_{\Delta}(\lambda)=1$ if $\lambda \in \Delta$, and $\chi_{\Delta}(\lambda)=0$ if $\lambda \notin \Delta$. 
Let $\mathcal{B}_{\theta \gamma}^{\alpha \beta}$ be a class of functions $F$ defined on $\sigma_{\alpha} \times \sigma_{\beta}, \alpha, \beta=1,2$, for each $\lambda \in \sigma_{\alpha}, \mu \in \sigma_{\beta}$ as operator $F(\lambda, \mu): \mathcal{G}_{\beta}(\mu) \rightarrow \mathcal{G}_{\alpha}(\lambda)$, with $\|F\|_{\mathcal{B}}<\infty$, where

$$
\begin{gathered}
\|F\|_{\mathcal{B}}=\sup _{\substack{\mu \in \sigma_{\beta} \\
\lambda \in \sigma_{\alpha}}}(1+|\lambda|)^{\theta}(1+|\mu|)^{\theta}|F(\lambda, \mu)|+ \\
+\sup _{\substack{\lambda, \lambda^{\prime} \in \sigma_{\alpha}^{c} \\
\mu \in \sigma_{\beta}}}\left\{(1+|\mu|)^{\theta} \frac{\left|F(\lambda, \mu)-F\left(\lambda^{\prime}, \mu\right)\right|}{\left|\lambda-\lambda^{\prime}\right|^{\gamma}}\right\}+ \\
+\sup _{\substack{\lambda \in \sigma_{\alpha} \\
\mu, \mu^{\prime} \in \sigma_{\beta}^{c}}}\left\{(1+|\lambda|)^{\theta} \frac{\left|F(\lambda, \mu)-F\left(\lambda, \mu^{\prime}\right)\right|}{\left|\mu-\mu^{\prime}\right|^{\gamma}}\right\}+ \\
+\sup _{\substack{\lambda, \lambda^{\prime} \in \sigma_{\alpha}^{c} \\
\mu, \mu^{\prime} \in \sigma_{\beta}^{c}}}\left\{\frac{\left|F(\lambda, \mu)-F\left(\lambda^{\prime}, \mu\right)-F\left(\lambda, \mu^{\prime}\right)+F\left(\lambda^{\prime}, \mu^{\prime}\right)\right|}{\left|\lambda-\lambda^{\prime}\right| \gamma\left|\mu-\mu^{\prime}\right|^{\gamma}}\right\} .
\end{gathered}
$$

With the norm $\|\cdot\|_{\mathcal{B}}$ this class will constitute a Banach space. We introduce also the Banach space $\mathcal{M}_{\theta \gamma}\left(\sigma_{\alpha}\right)$ of functions $f$ defined on $\sigma_{\alpha}$ with the norm

$$
\|f\|_{\mathcal{M}}=\sup _{\lambda \in \sigma_{\alpha}}(1+|\lambda|)^{\theta}|f(\lambda)|+\sup _{\lambda, \lambda^{\prime} \in \sigma_{\alpha}^{c}} \frac{\left|f(\lambda)-f\left(\lambda^{\prime}\right)\right|}{\left|\lambda-\lambda^{\prime}\right|^{\gamma}}<\infty .
$$

The value $f(\lambda)$ of the function $f \in \mathcal{M}_{\theta \gamma}\left(\sigma_{\alpha}\right)$ is an operator in $\mathcal{G}_{\alpha}(\lambda)$.

Let $B_{\alpha \beta}$ be an integral operator with a kernel $B_{\alpha \beta}(\lambda, \mu)$ from the space $\mathcal{B}_{\theta \gamma}^{\alpha \beta}, \theta>\frac{1}{2}, \frac{1}{2}<$ $\gamma<1$. We assume that $B_{\alpha \beta}(\lambda, \mu)$ is a compact operator, $B_{\alpha \beta}(\lambda, \mu): \mathcal{G}_{\beta}(\mu) \rightarrow \mathcal{G}_{\alpha}(\lambda)$, for each $\lambda \in \sigma_{\alpha}, \mu \in \sigma_{\beta}$ and $B_{\alpha \beta}(\lambda, \mu)=0$ if $\lambda$ belongs to the boundary of $\sigma_{\alpha}^{c}$ or $\mu$ belongs to the boundary of $\sigma_{\beta}^{c}$.

With this presuppositions the Hamiltonian $\mathbf{H}$ may be considered as a two-channel variant of the Friedrichs model [20], [21]. Investigation of $\mathbf{H}$ repeats almost literally the analysis from Ref. [21]. Therefore we describe here only final results which are quite analogous to [20], [21]. These results are following.

The operator $\mathbf{H}$ is self-adjoint on the set $\mathcal{D}(\mathbf{H})=\mathcal{D}\left(A_{1}\right) \oplus \mathcal{D}\left(A_{2}\right)$. Continuous spectrum of $\mathbf{H}$ is situated on the set $\sigma_{c}(\mathbf{H})=\sigma_{1}^{c} \cup \sigma_{2}^{c}$. Let $\mathbf{H}^{c}$ be the part of $\mathbf{H}$ acting in the invariant subspace corresponding to continuous spectrum. The operator $\mathbf{H}^{c}$ is unitary equivalent to the operator $\mathbf{H}_{0}=A_{1}^{(0)} \oplus A_{2}^{(0)}$ with $A_{\alpha}^{(0)}, \alpha=1,2$, the restriction of the operator $A_{\alpha}$ on $\mathcal{H}_{\alpha}^{c}$. Namely, there exist wave operators $U^{(+)}$and $U^{(-)}, U^{( \pm)}=\left(\begin{array}{ll}u_{11}^{( \pm)} & u_{12}^{( \pm)} \\ u_{21}^{( \pm)} & u_{22}^{( \pm)}\end{array}\right)=$ $s-\lim _{t \rightarrow \mp \infty} \mathrm{e}^{i \mathbf{H} t} \mathrm{e}^{-i \mathbf{H}_{0} t}$, with the following properties: $\mathbf{H} U^{( \pm)}=U^{( \pm)} \mathbf{H}_{0}, U^{( \pm) *} U^{( \pm)}=I$, $U^{( \pm)} U^{( \pm) *}=I-P$. Here, $P$ is an orthogonal projector on subspace corresponding to the discrete spectrum $\sigma_{d}(\mathbf{H})$ of the operator $\mathbf{H}$.

The kernel $u_{\alpha \alpha}^{( \pm)}\left(\lambda, \lambda^{\prime}\right)$ of the operator $u_{\alpha \alpha}^{( \pm)}, \alpha=1,2$, represents an eigenfunction of the continuous spectrum of the problem (2) for $z=\lambda^{\prime} \pm i 0, \lambda^{\prime} \in \sigma_{\alpha}^{c}$, and satisfies the integral equation

$$
u_{\alpha \alpha}^{( \pm)}\left(\lambda, \lambda^{\prime}\right)=I_{\alpha}^{c} \delta\left(\lambda-\lambda^{\prime}\right)-\left[\left(A_{\alpha}-\lambda^{\prime} \mp i 0\right)^{-1} V_{\alpha}\left(\lambda^{\prime} \pm i 0\right) u_{\alpha \alpha}^{( \pm)}\right]\left(\lambda, \lambda^{\prime}\right),
$$


where $I_{\alpha}^{c}$ is identity operator in $\mathcal{G}_{\alpha}^{c}, \lambda \in \sigma_{\alpha}$. For each concrete sign (plus or minus) and for each $\lambda^{\prime} \in \sigma_{\alpha}^{c}, \lambda^{\prime} \notin \sigma_{d}(\mathbf{H})$ the function $u_{\alpha \alpha}^{( \pm)}\left(\lambda, \lambda^{\prime}\right)$ is an unique solution of eq.(9) in the class of the distributions

$$
f_{\alpha}^{( \pm)}(\lambda)=I_{\alpha} \delta\left(\lambda-\lambda^{\prime}\right)+\frac{f(\lambda)}{\lambda-\lambda^{\prime} \mp i 0}, \quad f \in \mathcal{M}_{\theta^{\prime} \gamma^{\prime}}
$$

where $\frac{1}{2}<\theta^{\prime}<\theta, \frac{1}{2}<\gamma^{\prime}<\gamma$. At the same time

$$
u_{\alpha \beta}^{( \pm)}\left(\lambda, \lambda^{\prime}\right)=-\left[\left(A_{\alpha}-\lambda^{\prime} \mp i 0\right)^{-1} B_{\alpha \beta} u_{\beta \beta}^{( \pm)}\right]\left(\lambda, \lambda^{\prime}\right), \quad \beta \neq \alpha,
$$

is the problem (2) eigenfunction corresponding to $\lambda^{\prime} \in \sigma_{\beta}^{c}$.

The functions $u_{\beta \alpha}^{( \pm)}, \alpha, \beta=1,2$, can be explicitly expressed in terms of kernels of the operator

$$
T(z)=B-B(\mathbf{H}-z)^{-1} B, \quad B=\left[\begin{array}{lr}
0 & B_{12} \\
B_{21} & 0
\end{array}\right] .
$$

Corresponding formulae read as

$$
u_{\beta \alpha}^{( \pm)}\left(\lambda, \lambda^{\prime}\right)=\delta_{\beta \alpha} I_{\alpha}^{c} \delta\left(\lambda-\lambda^{\prime}\right)-\frac{T_{\beta \alpha}\left(\mu, \lambda^{\prime}, \lambda^{\prime} \pm i 0\right)}{\mu-\lambda^{\prime} \mp i 0}, \quad \mu \in \sigma_{\beta}, \quad \lambda^{\prime} \in \sigma_{\alpha}^{c}
$$

with $t$-matrices

$$
T_{\alpha \alpha}=B_{\alpha \beta}\left[z-A_{\beta}+B_{\beta \alpha}\left(A_{\alpha}-z\right)^{-1} B_{\alpha \beta}\right]^{-1} B_{\beta \alpha}
$$

and

$$
\begin{aligned}
& T_{\beta \alpha}=B_{\beta \alpha}\left[z-A_{\alpha}+B_{\alpha \beta}\left(A_{\beta}-z\right)^{-1} B_{\beta \alpha}\right]^{-1}\left(z-A_{\alpha}\right)= \\
= & \left(z-A_{\beta}\right)\left[z-A_{\beta}+B_{\beta \alpha}\left(A_{\alpha}-z\right)^{-1} B_{\alpha \beta}\right]^{-1} B_{\beta \alpha}, \quad \beta \neq \alpha .
\end{aligned}
$$

Considering the equation for $T(z), T(z)=B-B(A-z)^{-1} T(z), A=A_{1} \oplus A_{2}$, one shows in the same way as in [20], [21] that for all $z \in \mathbf{C} \backslash \sigma(\mathbf{H})$, each kernel $T_{\beta \alpha}(\mu, \lambda, z), \alpha, \beta=1,2$, belongs to the class $\mathcal{B}_{\theta^{\prime} \gamma^{\prime}}^{\beta \alpha}$ with arbitrary $\theta^{\prime}, \gamma^{\prime}$ such that $\frac{1}{2}<\theta^{\prime}<\theta, \frac{1}{2}<\gamma^{\prime}<\gamma$. In respect with variable $z$, the kernel of $T_{\beta \alpha}(z)$ is continuous in the $\mathcal{B}_{\theta^{\prime} \gamma^{\prime}}^{\beta \alpha}-$ norm right up to the upper and lower borders of the set $\sigma_{c}(\mathbf{H}) \backslash \sigma_{d}(\mathbf{H})$.

Scattering operator $S=U^{(-) *} U^{(+)}$for a system described by the Hamiltonian $\mathbf{H}$ is unitary in $\mathcal{H}_{\alpha}^{c}$. It's kernels $s_{\beta \alpha}(\mu, \lambda), \alpha, \beta=1,2$, are given by expressions

$$
s_{\beta \alpha}(\mu, \lambda)=\delta(\mu-\lambda)\left[\delta_{\beta \alpha} I_{\alpha}^{c}-2 \pi i T_{\beta \alpha}(\mu, \lambda, \lambda+i 0)\right] .
$$

By $U_{j}, j=1,2, \ldots$, we denote eigenvectors, $U_{j}=\left\{u_{1}^{(j)}, u_{2}^{(j)}\right\}, U_{j} \in \mathcal{D}(\mathbf{H}),\left\|U_{j}\right\|=1$, and by $z_{j}, z_{j} \in \mathbf{R}$, the respective eigenvalues of the operator $\mathbf{H}$ discrete spectrum $\sigma_{d}(\mathbf{H})$. The component $u_{\alpha}^{(j)}, \alpha=1,2$, of the vector $U_{j}$ is a solution of Eq. (2) at $z=z_{j}$. If $z_{j} \in \sigma_{\beta}^{c}$ then $\left(B_{\beta \alpha} u_{\alpha}^{(j)}\right)\left(z_{j}\right)=0$. 


\section{CONSTRUCTION OF THE OPERATORS $H_{\alpha}$}

The paper is devoted to construction of such operator $H_{\alpha}$ that it's each eigenfunction $u_{\alpha}, H_{\alpha} u_{\alpha}=z u_{\alpha}$, together with eigenvalue $z$, satisfies Eq. (2). This operator will be found as a solution of the non-linear operator equation (4). To obtain this equation we need the following operator-value function $V_{\alpha}(Y)$ of the operator variable $Y$ :

$$
V_{\alpha}(Y)=B_{\alpha \beta} \int_{\sigma_{\beta}} E_{\beta}(d \mu) B_{\beta \alpha}(Y-\mu)^{-1}
$$

$Y: \mathcal{H}_{\alpha} \rightarrow \mathcal{H}_{\alpha}$. We suppose here that $(Y-\mu I)^{-1} \in L_{\infty}\left(\sigma_{\beta}, \mathbf{B}\left(\mathcal{H}_{\alpha}, \mathcal{H}_{\alpha}\right)\right)$ if $\mu \in \sigma_{\beta}$. This means that $\sigma_{\beta}$ has not to be included into the spectrum of the operator $Y$. The integral $Q(T)=\int_{\sigma_{\beta}} E_{\beta}(d \mu) B_{\beta \alpha} T(\mu)$ may be constructed in the same way as integrals of scalar functions over spectral measure [27]. Namely, we consider $Q(T)$ for $T \in L_{\infty}\left(\sigma_{\beta}, \mathbf{B}\left(\mathcal{H}_{\alpha}, \mathcal{H}_{\alpha}\right)\right)$, $\|T\|_{\infty}=E_{\beta}-\sup _{\mu \in \sigma_{\beta}}\|T(\mu)\|<\infty$ (hereafter $E_{\beta}$-sup means the supremum with respect to $E_{\beta}$ ), as a limit value, with respect to the operator norm in $\mathbf{B}\left(\mathcal{H}_{\alpha}, \mathcal{H}_{\alpha}\right)$, of finite integral sums for piecewise-constant operator-value functions approximating $T$ in $L_{\infty}\left(\sigma_{\beta}, \mathbf{B}\left(\mathcal{H}_{\alpha}, \mathcal{H}_{\alpha}\right)\right)$. We show the existence of this integral at least in the case where the Hilbert-Schmidt norm $\left\|B_{\alpha \beta}\right\|_{2}$ is finite.

Lemma 1 Let $T \in L_{\infty}\left(\sigma_{\beta}, \mathbf{B}\left(\mathcal{H}_{\alpha}, \mathcal{H}_{\alpha}\right)\right)$ and $\left\|B_{\alpha \beta}\right\|_{2}<\infty$. Then the integral $Q(T)$ exists as a bounded operator in $\mathcal{H}_{\alpha}$ with norm satisfying the estimate $\|Q(T)\| \leq\|T\|_{\infty} \cdot\left\|B_{\beta \alpha}\right\|_{2}$.

Proof. We prove the Lemma in the diagonal representation (6), (7). By (8) we have

$$
(Q f)(\mu)=£_{\sigma_{\alpha}} B_{\beta \alpha}(\mu, \lambda)(T(\mu) f)(\lambda) d \lambda
$$

for any $f \in \mathcal{H}_{\alpha}$. It means that

$$
\begin{gathered}
|(Q f)(\mu)|^{2} \leq \sum_{\lambda \in \sigma_{\alpha}} d \lambda \cdot\left|B_{\beta \alpha}(\mu, \lambda)\right|^{2} \sum_{\lambda \in \sigma_{\alpha}} d \lambda|(T(\mu) f)(\lambda)|^{2}= \\
=\sum_{\lambda \in \sigma_{\alpha}} d \lambda\left|B_{\beta \alpha}(\mu, \lambda)\right|^{2} \cdot\|T(\mu) f\|^{2} \leq \sum_{\lambda \in \sigma_{\alpha}} d \lambda\left|B_{\beta \alpha}(\mu, \lambda)\right|^{2} \cdot\|T(\mu)\|^{2} \cdot\|f\|^{2} .
\end{gathered}
$$

Hence, integrating over $\mu \in \sigma_{\beta}$ we come to the relation

$$
\|Q f\|^{2} \leq\left\|B_{\beta \alpha}\right\|_{2}^{2} \cdot\|T\|_{\infty}^{2} \cdot\|f\|^{2}
$$

which completes the proof.

Let us suppose that $\left(H_{\alpha}-\mu I\right)^{-1} \in L_{\infty}\left(\sigma_{\beta}, \mathbf{B}\left(\mathcal{H}_{\alpha}, \mathcal{H}_{\alpha}\right)\right)$. We note that if $H_{\alpha} \psi_{\alpha}=z \psi_{\alpha}$, then automatically

$$
V_{\alpha}\left(H_{\alpha}\right) \psi_{\alpha}=B_{\alpha \beta} \int_{\sigma_{\beta}} E_{\beta}(d \mu) B_{\beta \alpha}(z-\mu)^{-1} \psi_{\alpha}=
$$




$$
=B_{\alpha \beta}\left(z-A_{\beta}\right)^{-1} B_{\beta \alpha} \psi_{\alpha}=V_{\alpha}(z) \psi_{\alpha} .
$$

It follows from (12) that $H_{\alpha}$ satisfies the relation $H_{\alpha} \psi_{\alpha}=\left(A_{\alpha}+V_{\alpha}\left(H_{\alpha}\right)\right) \psi_{\alpha}$ and we can spread this relation over all the linear combinations of $H_{\alpha}$ eigenfunctions. Supposing that the eigenfunctions system of $H_{\alpha}$ is dense in $\mathcal{H}_{\alpha}$ we spread this equation over $\mathcal{D}\left(A_{\alpha}\right)$. As a result we come to the desired basic equation (14) for $H_{\alpha}$ (see also Refs. [9], [17]- [19]). Eq. (14) means that the construction of the operator $H_{\alpha}$ comes to the searching for the operator

$$
Q_{\beta \alpha}=\int_{\sigma_{\beta}} E_{\beta}(d \mu) B_{\beta \alpha}\left(H_{\alpha}-\mu\right)^{-1} .
$$

Since $H_{\alpha}=A_{\alpha}+B_{\alpha \beta} Q_{\beta \alpha}$, we have

$$
Q_{\beta \alpha}=\int_{\sigma_{\beta}} E_{\beta}(d \mu) B_{\beta \alpha}\left(A_{\alpha}+B_{\alpha \beta} Q_{\beta \alpha}-\mu\right)^{-1}, \quad \beta \neq \alpha .
$$

In this paper we restrict ourselves to the study of Eq. (14) solvability only in the case where spectra $\sigma_{1}$ and $\sigma_{2}$ are separated,

$$
d_{0}=\operatorname{dist}\left(\sigma_{1}, \sigma_{2}\right)>0 .
$$

Using the Lemma 1 and the contracting mapping Theorem, we prove the following:

Theorem 1 Let $M_{\beta \alpha}(\delta)$ be a set of bounded operators $X, X: \mathcal{H}_{\alpha} \rightarrow \mathcal{H}_{\beta}$, satisfying the inequality $\|X\| \leq \delta$ with $\delta>0$. If this $\delta$ and the norm $\left\|B_{\alpha \beta}\right\|_{2}$ satisfy the condition $\left\|B_{\alpha \beta}\right\|_{2}<$ $d_{0} \min \left\{\frac{1}{1+\delta}, \frac{\delta}{1+\delta^{2}}\right\}$, then $E q$. (14) is uniquely solvable in $M_{\beta \alpha}(\delta)$.

Proof. Let

$$
F(X)=\int_{\sigma_{\beta}} E_{\beta}(d \mu) B_{\beta \alpha}\left(A_{\alpha}+B_{\alpha \beta} X-\mu\right)^{-1}
$$

with $X$, the operator from $\mathbf{B}\left(\mathcal{H}_{\alpha}, \mathcal{H}_{\beta}\right)$.

Firstly, consider conditions where the function $F$ maps the set $M_{\alpha}(\delta)$ into itself. We suppose here that $B_{\alpha \beta}$ and $X$ are such that

$$
\left\|B_{\alpha \beta}\right\|_{2}\|X\| \leq \delta\left\|B_{\alpha \beta}\right\|_{2}<d_{0}
$$

and consequently, $\left\|B_{\alpha \beta} X\right\| \leq d_{0}$. This means that spectrum of the operator $A_{\alpha}+B_{\alpha \beta} X$ does not intersect with the set $\sigma_{\beta}$. Hence, the resolvent $\left(A_{\alpha}+B_{\alpha \beta} X-\mu\right)^{-1}$ exists and is bounded for any $\mu \in \sigma_{\beta}$. Thus, by Lemma 1 we have

$$
\|F(X)\| \leq\left\|B_{\alpha \beta}\right\|_{2} \cdot E_{\beta}-\sup _{\mu \in \sigma_{\beta}}\left\|\left(A_{\alpha}+B_{\alpha \beta} X-\mu\right)^{-1}\right\| .
$$

Due to identity

$$
\left(A_{\alpha}+B_{\alpha \beta} X-\mu\right)^{-1}=\left(I+\left(A_{\alpha}-\mu\right)^{-1} B_{\alpha \beta} X\right)^{-1}\left(A_{\alpha}-\mu\right)^{-1}
$$


and inequality $\left\|B_{\alpha \beta}\right\| \leq\left\|B_{\alpha \beta}\right\|_{2}$ we make estimation

$$
\begin{gathered}
\left\|\left(A_{\alpha}+B_{\alpha \beta} X-\mu\right)^{-1}\right\| \leq \frac{1}{1-\left\|\left(A_{\alpha}-\mu\right)^{-1}\right\|\left\|B_{\alpha \beta}\right\|_{2}\|X\|}\left\|\left(A_{\alpha}-\mu\right)^{-1}\right\| \leq \\
\leq \frac{1}{1-\frac{1}{d_{0}}\left\|B_{\alpha \beta}\right\|_{2} \delta} \cdot \frac{1}{d_{0}}=\frac{1}{d_{0}-\left\|B_{\alpha \beta}\right\|_{2} \delta} .
\end{gathered}
$$

Therefore, the set $M_{\alpha}(\delta)$ will be mapped by $F$ into itself if $\left\|B_{\alpha \beta}\right\|_{2}$ and $\delta$ are such that

$$
\left\|B_{\alpha \beta}\right\|_{2} \cdot \frac{1}{d_{0}-\left\|B_{\alpha \beta}\right\|_{2} \delta} \leq \delta .
$$

Secondly, study conditions for the function $F$ to be a contracting mapping. Now, we consider the difference

$$
\begin{gathered}
F(X)-F(Y)=\int_{\sigma_{\beta}} E_{\beta}(d \mu) B_{\beta \alpha}\left[\left(A_{\alpha}+B_{\alpha \beta} X-\mu\right)^{-1}-\left(A_{\alpha}+B_{\alpha \beta} Y-\mu\right)^{-1}\right]= \\
=\int_{\sigma_{\beta}} E_{\beta}(d \mu) B_{\beta \alpha}\left(A_{\alpha}+B_{\alpha \beta} X-\mu\right)^{-1} B_{\alpha \beta}(Y-X)\left(A_{\alpha}+B_{\alpha \beta} Y-\mu\right)^{-1} .
\end{gathered}
$$

Again, by Lemma 1, we have

$$
\begin{gathered}
\|F(X)-F(Y)\| \leq \\
\leq\left\|B_{\alpha \beta}\right\|_{2}^{2} \cdot \sup _{\mu \in \sigma_{\beta}}\left\|\left(A_{\alpha}+B_{\alpha \beta} X-\mu\right)^{-1}\right\| \cdot \sup _{\mu \in \sigma_{\beta}}\left\|\left(A_{\alpha}+B_{\alpha \beta} Y-\mu\right)^{-1}\right\| \cdot\|(Y-X)\| .
\end{gathered}
$$

With (18) we come to the estimate

$$
\|F(X)-F(Y)\| \leq\left\|B_{\alpha \beta}\right\|_{2}^{2} \cdot \frac{1}{\left(d_{0}-\left\|B_{\alpha \beta}\right\|_{2} \delta\right)^{2}} \cdot\|Y-X\| .
$$

The function $F$ becomes a contracting mapping if

$$
\frac{\left\|B_{\alpha \beta}\right\|_{2}^{2}}{\left(d_{0}-\left\|B_{\alpha \beta}\right\|_{2} \delta\right)^{2}}<1 .
$$

Solving system of the inequalities (17), (19) and (20) we find

$$
\left\|B_{\alpha \beta}\right\|_{2}<d_{0} \min \left\{\frac{\delta}{1+\delta^{2}}, \frac{1}{1+\delta}\right\}
$$

and this completes the proof of Theorem 1 .

Corollary 1 Equation (14) is uniquely solvable in the unit ball $M_{\beta \alpha}(1) \subset \mathbf{B}\left(\mathcal{H}_{\alpha}, \mathcal{H}_{\beta}\right)$ for any $B_{\alpha \beta}$ such that

$$
\left\|B_{\alpha \beta}\right\|_{2}<\frac{1}{2} d_{0} .
$$


To prove the inequality (21), note that $\max _{\delta \geq 0} \min \left\{\frac{\delta}{1+\delta^{2}}, \frac{1}{1+\delta}\right\}=\frac{1}{2}$ (at $\delta=1$ ). Hence, if (21) takes place then the function (16) is a contracting mapping of the unit ball $M_{\beta \alpha}(1)$ into itself.

Remark. In the proofs of Lemma 1 and Theorem 1 we did not use the assumption about finiteness of the numbers $n_{\alpha}$ of intervals included in continuous spectra $\sigma_{\alpha}^{c}$ of the operators $A_{\alpha}, \alpha=1,2$. Really, these assertions take place in the case of arbitrary spectrum $\sigma_{\alpha}$.

Finiteness at least of one of the numbers $n_{1}$ and $n_{2}$ will be used at the moment. If $n_{1}$ and/or $n_{2}$ are finite and

$$
\left\|B_{\alpha \beta} Q_{\beta \alpha}\right\|<d_{0}=\operatorname{dist}\left\{\sigma_{1}, \sigma_{2}\right\}, \quad \alpha=1,2, \beta \neq \alpha,
$$

we can state that

$$
\left\|\left(A_{\alpha}+B_{\alpha \beta} Q_{\beta \alpha}-\mu\right)^{-1}\right\| \leq \frac{C_{\alpha \beta}}{1+|\mu|}, \alpha=1,2, \quad \text { at any } \mu \in \sigma_{\beta}, \beta \neq \alpha,
$$

with some $C_{\alpha \beta}>0, C_{\alpha \beta} \sim 1 /\left(d_{0}-\left\|B_{\alpha \beta} Q_{\beta \alpha}\right\|\right)$. Of course this estimate is essential only in the case where $\sigma_{\beta}$ is unbounded. It follows immediately from Eq. (14) that if $n_{1}$ and/or $n_{2}$ are finite then $Q_{\beta \alpha} f_{\alpha} \in \mathcal{D}\left(H_{\beta}\right)=\mathcal{D}\left(A_{\beta}\right)$ for any $f_{\alpha} \in \mathcal{H}_{\alpha}$.

In this case we can rewrite Eq. (14) in symmetric form as a stationary Riccati equation (see the book [28] and Refs. therein),

$$
Q_{\beta \alpha} A_{\alpha}-A_{\beta} Q_{\beta \alpha}+Q_{\beta \alpha} B_{\alpha \beta} Q_{\beta \alpha}=B_{\beta \alpha} .
$$

To make this, it is sufficient to calculate the expression $Q_{\beta \alpha} H_{\alpha}-A_{\beta} Q_{\beta \alpha}$ for both parts of eq.(14) having in mind that we apply it to $f_{\alpha} \in \mathcal{D}\left(H_{\alpha}\right)$. Did, we have

$$
Q_{\beta \alpha} H_{\alpha}-A_{\beta} Q_{\beta \alpha}=Q_{\beta \alpha}\left(A_{\alpha}+B_{\alpha \beta} Q_{\beta \alpha}\right)-A_{\beta} Q_{\beta \alpha}=Q_{\beta \alpha} A_{\alpha}-A_{\beta} Q_{\beta \alpha}+Q_{\beta \alpha} B_{\alpha \beta} Q_{\beta \alpha} .
$$

On the other hand,

$$
Q_{\beta \alpha} H_{\alpha}-A_{\beta} Q_{\beta \alpha}=\int_{\sigma_{\beta}}\left[E_{\beta}(d \mu) B_{\beta \alpha}\left(H_{\alpha}-\mu\right)^{-1} H_{\alpha}-\mu E_{\beta}(d \mu) B_{\beta \alpha}\left(H_{\alpha}-\mu\right)^{-1}\right]=B_{\beta \alpha} .
$$

One finds immediately from Eqs. (24), $\alpha=1,2$, that if $Q_{\beta \alpha}$ gives solution $H_{\alpha}=A_{\alpha}+B_{\alpha \beta} Q_{\beta \alpha}$ of the problem (国) in the channel $\alpha$ then

$$
Q_{\alpha \beta}=-Q_{\beta \alpha}^{*}=-\int_{\sigma_{\alpha}}\left(H_{\alpha}^{*}-\mu\right)^{-1} B_{\alpha \beta} E_{\beta}(d \mu)
$$

gives analogous solution $H_{\beta}=A_{\beta}+B_{\beta \alpha} Q_{\alpha \beta}$ in the channel $\beta$.

Theorem 2 Let $Q_{\beta \alpha}, Q_{\beta \alpha} \in \mathbf{B}\left(\mathcal{H}_{\alpha}, \mathcal{H}_{\beta}\right)$, be a solution of Eq. (24) satisfying together with $Q_{\alpha \beta}=-Q_{\beta \alpha}^{*}$ the conditions (23). Then the transform $\mathbf{H}^{\prime}=\mathcal{Q}^{-1} \mathbf{H} \mathcal{Q}$ with $\mathcal{Q}=\left[\begin{array}{rr}I_{1} & Q_{12} \\ Q_{21} & I_{2}\end{array}\right]$ reduces the operator $\mathbf{H}$ to the block-diagonal form, $\mathbf{H}^{\prime}=\operatorname{diag}\left\{H_{1}, H_{2}\right\}$ where $H_{\alpha}=A_{\alpha}+$ $B_{\alpha \beta} Q_{\beta \alpha}, \alpha, \beta=1,2, \beta \neq \alpha$. At the same time, the operators $\mathcal{O}_{\alpha}=\left[\begin{array}{lr}I_{\alpha} & 0 \\ Q_{\beta \alpha} & I_{\beta}\end{array}\right]$ reduce the Hamiltonian $\mathbf{H}, \mathbf{H}=\left[\begin{array}{cr}A_{\alpha} & B_{\alpha \beta} \\ B_{\beta \alpha} & A_{\beta}\end{array}\right]$, to triangular form, $\mathbf{H}^{(\alpha)} \equiv \mathcal{O}_{\alpha}^{-1} \mathbf{H} \mathcal{O}_{\alpha}=\left[\begin{array}{cc}H_{\alpha} & B_{\alpha \beta} \\ 0 & H_{\beta}^{*}\end{array}\right]$. 
Proofs of both statements are done by direct substituting $\mathcal{Q}$ and $\mathcal{O}_{\alpha}$ into the definitions of $\mathbf{H}^{\prime}$ and $\mathbf{H}^{(\alpha)}$ and using the equations (24).

We have to note only that operator $\mathcal{Q}$ is invertible since, according to (25),

$$
X_{\alpha}=I_{\alpha}-Q_{\alpha \beta} Q_{\beta \alpha}=I_{\alpha}+Q_{\alpha \beta} Q_{\alpha \beta}^{*} \geq I_{\alpha}, \quad \alpha=1,2
$$

and

$$
\mathcal{Q}^{-1}=\left[\begin{array}{lr}
X_{1}^{-1} & 0 \\
0 & X_{2}^{-1}
\end{array}\right] \cdot\left[\begin{array}{lr}
I_{1} & -Q_{12} \\
-Q_{21} & I_{2}
\end{array}\right]
$$

Corollary 2 Subspaces $\mathcal{H}^{(\alpha)}=\mathcal{O}_{\alpha}\left(\mathcal{H}_{\alpha} \oplus\{\mathbf{0}\}\right)=\left\{f: f=\left\{f_{\alpha}, f_{\beta}\right\} \in \mathcal{H}, f_{\alpha} \in \mathcal{H}_{\alpha}, f_{\beta}=\right.$ $\left.Q_{\beta \alpha} f_{\alpha}\right\}$ are orthogonal, $\mathcal{H}^{(1)} \perp \mathcal{H}^{(2)}$, and reducing for $\mathbf{H}, \mathbf{H}\left(\mathcal{D}(\mathbf{H}) \cap \mathcal{H}^{(\alpha)}\right) \subseteq \mathcal{H}^{(\alpha)}$.

Really, if $f \in \mathcal{H}^{(\alpha)}, g \in \mathcal{H}^{(\beta)}$ and $f=\left\{f_{\alpha}, Q_{\beta \alpha} f_{\alpha}\right\}, g=\left\{Q_{\alpha \beta} g_{\beta}, g_{\beta}\right\}$, then $\langle f, g\rangle=$ $\left\langle f_{\alpha}, Q_{\alpha \beta} g_{\beta}\right\rangle+\left\langle Q_{\beta \alpha} f_{\alpha}, g_{\beta}\right\rangle=0$ since $Q_{\beta \alpha}=-Q_{\alpha \beta}^{*}$. The invariance of $\mathcal{H}^{(\alpha)}, \alpha=1,2$, in respect with $\mathbf{H}$ follows from the equality $\mathbf{H} \mathcal{Q}=\mathcal{Q} \mathbf{H}^{\prime}$.

Assertions quite analogous to the Theorem 2 and Corollary 2 one can find in Refs. [22], [23]. Solvability (for sufficiently small $\left\|B_{\alpha \beta}\right\|$ ) of the equation (24) was proved in [22], [23] by rather different method also in the supposition (15).

Remark. It follows from Theorem 2 that operator $\tilde{\mathcal{Q}}=\mathcal{Q} X^{-1 / 2}$ with $X=\operatorname{diag}\left\{X_{1}, X_{2}\right\}$ is unitary. Consequently, the operator $\mathbf{H}^{\prime \prime}=\tilde{\mathcal{Q}}^{*} \mathbf{H} \tilde{\mathcal{Q}}=X^{1 / 2} \mathbf{H}^{\prime} X^{-1 / 2}$ becomes self-adjoint in $\mathcal{H}$. Since $\mathbf{H}^{\prime \prime}=\operatorname{diag}\left\{H_{1}^{\prime \prime}, H_{1}^{\prime \prime}\right\}$ with $H_{\alpha}^{\prime \prime}=X_{\alpha}^{1 / 2} H_{\alpha} X_{\alpha}^{-1 / 2}$, the operators $H_{\alpha}^{\prime \prime}, \quad \alpha=1,2$, are self-adjoint on $\mathcal{D}\left(A_{\alpha}\right)$ in $\mathcal{H}_{\alpha}$. Moreover the operators $\mathbf{H}^{(\alpha)}=\tilde{\mathcal{Q}} \cdot \operatorname{diag}\left\{H_{\alpha}^{\prime \prime}, 0\right\} \cdot \tilde{\mathcal{Q}}^{*}=$ $\mathcal{Q} \cdot \operatorname{diag}\left\{H_{\alpha}, 0\right\} \cdot \mathcal{Q}^{-1}$ represent parts of the Hamiltonian $\mathbf{H}$ in the corresponding invariant subspaces $\mathcal{H}^{(1)}$ and $\mathcal{H}^{(2)}$ (see also Refs. [22], [23]).

Unfortunately, eigenvectors $\psi_{\alpha}^{\prime \prime}$ of the operators $H_{\alpha}^{\prime \prime}$ differ from those for the initial spectral problem (2): $\psi^{\prime \prime}{ }_{\alpha}=X_{\alpha}^{1 / 2} \psi_{\alpha}$.

Lemma 2 Let the kernel $B_{\beta \alpha}(\mu, \lambda), \beta \neq \alpha$, of the operator $B_{\beta \alpha}$ belong to the class $\mathcal{B}_{\theta \gamma}^{\beta \alpha}$ with $\theta>\frac{1}{2}$ and $Q_{\beta \alpha}$ be a solution of Eq. (14) satisfying together with $Q_{\alpha \beta}=-Q_{\beta \alpha}^{*}$ the conditions (23). Then

(a) the operator $Q_{\beta \alpha}$ is an integral operator, $Q_{\beta \alpha}: \mathcal{H}_{\alpha} \rightarrow \mathcal{H}_{\beta}$, with a kernel $Q_{\beta \alpha}(\mu, \lambda)$ belonging to $\mathcal{B}_{\theta \gamma}^{\beta \alpha}$;

(b) the potential $W_{\alpha} \equiv B_{\alpha \beta} Q_{\beta \alpha}$ is an integral operator, $W_{\alpha}: \mathcal{H}_{\alpha} \rightarrow \mathcal{H}_{\alpha}$, with a kernel $W_{\alpha}\left(\lambda, \lambda^{\prime}\right)$ belonging to $\mathcal{B}_{\theta \gamma}^{\alpha \alpha}$.

Proof. At the beginning we prove the assertion (b). According to (25),

$$
W_{\alpha}=-B_{\alpha \beta} \int_{\sigma_{\alpha}}\left(H_{\beta}^{*}-\lambda\right)^{-1} B_{\beta \alpha} E_{\alpha}(d \lambda)
$$

with $H_{\beta \alpha}^{*}=A_{\beta}+W_{\beta}^{*}=A_{\beta}+Q_{\alpha \beta}^{*} B_{\alpha \beta}$. Since the inequalities (23) take place we write

$$
\left\|\left(H_{\beta}^{*}-\lambda\right)^{-1}\right\|=\left\|\left(H_{\beta}-\lambda\right)^{-1}\right\| \leq C_{\beta \alpha}
$$


for any $\lambda \in \sigma_{\alpha}$. In the diagonal representation (6),(17), the equation (28) turns in

$$
W_{\alpha}\left(\lambda, \lambda^{\prime}\right)=-B_{\alpha \beta}(\lambda, \cdot)\left(H_{\beta}^{*}-\lambda\right)^{-1} B_{\beta \alpha}\left(\cdot, \lambda^{\prime}\right) .
$$

It means that

$$
\begin{aligned}
\left|W_{\alpha}\left(\lambda, \lambda^{\prime}\right)\right| & \leq\left\|B_{\alpha \beta}(\lambda, \cdot)\right\|_{\mathcal{H}_{\beta}} \cdot\left\|\left(H_{\beta}^{*}-\lambda\right)^{-1}\right\| \cdot\left\|B_{\beta \alpha}\left(\cdot, \lambda^{\prime}\right)\right\|_{\mathcal{H}_{\beta}} \leq \\
& \leq C_{\beta \alpha}\left\|B_{\alpha \beta}(\lambda, \cdot)\right\|_{\mathcal{H}_{\beta}} \cdot\left\|B_{\beta \alpha}\left(\cdot, \lambda^{\prime}\right)\right\|_{\mathcal{H}_{\beta}} .
\end{aligned}
$$

Here, $\left\|B_{\alpha \beta}(\lambda, \cdot)\right\|_{\mathcal{H}_{\beta}}=\left[\oint_{\sigma_{\beta}}\left|B_{\alpha \beta}(\lambda, \mu)\right|^{2} d \mu\right]^{1 / 2}$. Since $\theta>\frac{1}{2}$, we have $\left\|B_{\alpha \beta}(\lambda, \cdot)\right\|_{\mathcal{H}_{\beta}} \leq \frac{c(\theta)}{(1+|\lambda|)^{\theta}}$. $\|B\|_{\mathcal{B}}$ with some $c(\theta), c(\theta)>0$, depending only on $\theta$. Analogously,

$$
\left\|B_{\beta \alpha}\left(\cdot, \lambda^{\prime}\right)\right\|_{\mathcal{H}_{\beta}}=\left\|\overline{B_{\alpha \beta}}\left(\lambda^{\prime}, \cdot\right)\right\|_{\mathcal{H}_{\beta}} \leq \frac{c(\theta)}{(1+|\lambda|)^{\theta}} \cdot\|B\|_{\mathcal{B}}
$$

where the operator $\overline{B_{\alpha \beta}}(\lambda, \mu), \overline{B_{\alpha \beta}}(\lambda, \mu): \mathcal{G}_{\beta}(\mu) \rightarrow \mathcal{G}_{\alpha}(\lambda)$, is adjoint to $B_{\beta \alpha}(\mu, \lambda)$.

Estimations similar to (29) may be done also for $\left|W_{\alpha}\left(\lambda^{\prime \prime}, \lambda^{\prime}\right)-W_{\alpha}\left(\lambda, \lambda^{\prime}\right)\right|, \lambda, \lambda^{\prime \prime} \in \sigma_{\alpha}^{c}, \lambda \in$ $\sigma_{\alpha},\left|W_{\alpha}\left(\lambda, \lambda^{\prime \prime \prime}\right)-W_{\alpha}\left(\lambda, \lambda^{\prime}\right)\right|, \lambda \in \sigma_{\alpha}, \lambda^{\prime \prime \prime}, \lambda^{\prime} \in \sigma_{\alpha}^{c}$, and $\mid W_{\alpha}\left(\lambda, \lambda^{\prime}\right)-W_{\alpha}\left(\lambda^{\prime \prime}, \lambda^{\prime}\right)-W_{\alpha}\left(\lambda, \lambda^{\prime \prime \prime}\right)+$ $W_{\alpha}\left(\lambda^{\prime \prime}, \lambda^{\prime \prime \prime}\right) \mid, \quad \lambda, \lambda^{\prime}, \lambda^{\prime \prime}, \lambda^{\prime \prime \prime} \in \sigma_{\alpha}^{c}$, in terms of the norms $\left\|B_{\alpha \beta}(\lambda, \cdot)-B_{\alpha \beta}\left(\lambda^{\prime \prime}, \cdot\right)\right\|_{\mathcal{H}_{\beta}}$ and $\left\|B_{\beta \alpha}\left(\cdot, \lambda^{\prime \prime \prime}\right)-B_{\beta \alpha}\left(\cdot, \lambda^{\prime}\right)\right\|_{\mathcal{H}_{\beta}}$. Estimating the latter through $\left\|B_{\alpha \beta}\right\|_{\mathcal{B}}$ we come to the inequality

$$
\left\|W_{\alpha}\right\|_{\mathcal{B}_{\theta \gamma}^{\alpha \alpha}} \leq c(\theta) C_{\beta \alpha} \cdot\left\|B_{\beta \alpha}\right\|_{\mathcal{B}_{\theta \gamma}^{\beta \alpha}}^{2}
$$

with $0<c(\theta)<\infty$. Therefore, we have proved the assertion (b).

To prove the statement (a) we note that according to (14),

$$
Q_{\beta \alpha}=\int_{\sigma_{\beta}} E_{\beta}(d \mu) B_{\beta \alpha}\left[\left(A_{\alpha}-\mu\right)^{-1}-\left(H_{\alpha}-\mu\right)^{-1} W_{\alpha}\left(A_{\alpha}-\mu\right)^{-1}\right]
$$

or, in the diagonal representation (6),(7),

$$
Q_{\beta \alpha}(\mu, \lambda)=\frac{B_{\beta \alpha}(\mu, \lambda)}{\lambda-\mu}-\frac{B_{\beta \alpha}(\mu, \cdot)\left(H_{\alpha}-\mu\right)^{-1} W_{\alpha}(\cdot, \lambda)}{\lambda-\mu} .
$$

Repeating literally the last part of the proof of the assertion (b) we come to the inequality

$$
\begin{gathered}
\left\|Q_{\beta \alpha}\right\|_{\mathcal{B}_{\theta \gamma}^{\beta \alpha}} \leq \sup _{\substack{\mu \in \sigma_{\beta} \\
\lambda \in \sigma_{\alpha}}} \frac{1}{|\lambda-\mu|} \cdot\left\{\left\|B_{\beta \alpha}\right\|_{\mathcal{B}_{\theta \gamma}^{\beta \alpha}}+\right. \\
\left.+c(\theta) \cdot\left\|B_{\beta \alpha}\right\|_{\mathcal{B}_{\theta \gamma}^{\beta \alpha}} \cdot \sup _{\mu \in \sigma_{\beta}}\left\|\left(H_{\alpha}-\mu\right)^{-1}\right\| \cdot\left\|W_{\alpha}\right\|_{\mathcal{B}_{\theta \gamma}^{\alpha \alpha}}\right\}, \quad 0<c(\theta)<+\infty .
\end{gathered}
$$

Consequently $Q_{\beta \alpha} \in \mathcal{B}_{\theta \gamma}^{\beta \alpha}$ and

$$
\left\|Q_{\beta \alpha}\right\|_{\mathcal{B}} \leq \frac{1}{d_{0}} \cdot\left\{\left\|B_{\beta \alpha}\right\|_{\mathcal{B}}+c(\theta) C_{\alpha \beta} C_{\beta \alpha} \cdot\left\|B_{\beta \alpha}\right\|_{\mathcal{B}}^{3}\right\}, \quad 0<c(\theta)<+\infty .
$$

This completes the proof of Lemma 2 .

Corollary 3 If $B_{\beta \alpha} \in \mathcal{B}_{\theta \gamma}^{\beta \alpha}, \theta>\frac{1}{2}$, then the solution of Eq. (14) described by Theorem 1 belongs to the class $\mathcal{B}_{\theta \gamma}^{\beta \alpha}$, too.

This statement is based on the fact that the mentioned solution satisfies automatically the conditions (22) and, hence, the conditions (23). 


\section{EIGENFUNCTIONS AND THE EXPANSION THEOREM}

In the preceding section, we have proved the existence (in the unit ball $M_{\beta \alpha}(1) \subset$ $\mathbf{B}\left(\mathcal{H}_{\alpha}, \mathcal{H}_{\beta}\right)$ ) of a solution $Q_{\beta \alpha}$ of the basic equation (14) only in the case where spectra $\sigma_{1}$, $\sigma_{2}$ of the operators $A_{1}, A_{2}$ are separated, $\operatorname{dist}\left\{\sigma_{1}, \sigma_{2}\right\}=d_{0}>0$, and $\left\|B_{12}\right\|_{2}=\left\|B_{21}\right\|_{2}<\frac{d_{0}}{2}$. May be, however, Eqs. (4) and (14) have solutions also in other cases. That is why we study the spectral properties of the operator $H_{\alpha}=A_{\alpha}+B_{\alpha \beta} Q_{\beta \alpha}$ not supposing that $\left\|B_{\alpha \beta}\right\|_{2}<\frac{d_{0}}{2}$ and using more general requirements (23) only, with $C_{\alpha \beta}$, some positive numbers, $\alpha, \beta=1,2$, $\beta \neq \alpha$. Of course, we assume again that the condition (15) takes place. Remember that the requirements (23) are sufficient for existence of the operators $V_{\alpha}\left(H_{\alpha}\right)$. As well, the equations (24) and (25) take place and the assertions of Theorem 2 and Lemma 2 are valid.

So, let us suppose that $Q_{\beta \alpha}$ and $Q_{\alpha \beta}=-Q_{\beta \alpha}^{*}$ are solutions of Eqs. (14) and (24) satisfying the conditions (23). It follows from Lemma 11 that $Q_{\beta \alpha} \in \mathbf{B}_{\beta \alpha}\left(\mathcal{H}_{\alpha}, \mathcal{H}_{\beta}\right)$ as well as $Q_{\alpha \beta} \in$ $\mathbf{B}_{\alpha \beta}\left(\mathcal{H}_{\beta}, \mathcal{H}_{\alpha}\right)$. If $B_{\beta \alpha} \in \mathcal{B}_{\theta \gamma}^{\beta \alpha}, \theta>\frac{1}{2}$, then, according to Lemma 2 , $Q_{\beta \alpha} \in \mathcal{B}_{\theta \gamma}^{\beta \alpha}$ and $Q_{\alpha \beta} \in \mathcal{B}_{\theta \gamma}^{\alpha \beta}$.

By Theorem 2, the operator $\mathbf{H}^{\prime}=\operatorname{diag}\left\{H_{1}, H_{2}\right\}$ is connected with the (self-adjoint) operator $\mathbf{H}$ by a similarity transform. Thus, the spectra $\sigma\left(H_{1}\right)$ and $\sigma\left(H_{2}\right)$ of the operators $H_{\alpha}, \alpha=1,2$, are real and $\sigma\left(H_{1}\right) \cup \sigma\left(H_{2}\right)=\sigma(\mathbf{H})$. Continuous spectrum $\sigma_{c}\left(H_{\alpha}\right)$ of the each operator $H_{\alpha}$ coincides with that of the operator $A_{\alpha}, \sigma_{c}\left(H_{\alpha}\right)=\sigma_{\alpha}^{c}$, since due to $\left\|B_{\alpha \beta}\right\|_{2}<+\infty$, the potential $W_{\alpha}=B_{\alpha \beta} Q_{\beta \alpha}$ is a compact operator. Since $\sigma_{1}^{c} \cap \sigma_{2}^{c}=\emptyset$ we have $\sigma_{c}\left(H_{1}\right) \cap \sigma_{c}\left(H_{2}\right)=\emptyset$. We show now that the discrete spectra $\sigma_{d}\left(H_{\alpha}\right), \alpha=1$, 2 , satisfy a similar condition.

Let us suppose that $\sigma_{d}\left(H_{\alpha}\right) \neq \emptyset, z \in \sigma_{d}\left(H_{\alpha}\right)$ and $\psi_{\alpha}$ is the corresponding eigenfunction of $H_{\alpha}, H_{\alpha} \psi_{\alpha}=z \psi_{\alpha}, \psi_{\alpha} \in \mathcal{D}\left(H_{\alpha}\right)=\mathcal{D}\left(A_{\alpha}\right)$. Then, according to construction of $H_{\alpha}$, we have $H_{\alpha} \psi_{\alpha}=\left(A_{\alpha}+V_{\alpha}\left(H_{\alpha}\right)\right) \psi_{\alpha}=\left(A_{\alpha}+V_{\alpha}(z)\right) \psi_{\alpha}=z \psi_{\alpha}$. Thus if $z \in \sigma_{d}\left(H_{\alpha}\right)$ then $z$ becomes automatically a point of the discrete spectrum of the initial spectral problem (2). At the same time $\psi_{\alpha}$ becomes it's eigenfunction.

Let us further denote the eigenfunctions of the operator $H_{\alpha}$ discrete spectrum by $\psi_{\alpha}^{(j)}$, $\psi_{\alpha}^{(j)}=u_{\alpha}^{(j)}$, keeping for them the same numeration as for eigenvectors of $U_{j}, U_{j}=\left\{u_{\alpha}^{(j)}, u_{\beta}^{(j)}\right\}$, of the Hamiltonian $\mathbf{H}, \mathbf{H} U_{j}=z_{j} U_{j}, z_{j} \in \sigma_{d}(\mathbf{H})$. We assume that in the case of multiple discrete eigenvalues, certain $z_{j}$ may be repeated in this numeration. By $\mathcal{U}^{d}$ we denote the set $\mathcal{U}^{d}=\left\{U_{j}, j=1,2, \ldots\right\}$ of all the eigenvectors $U_{j}$.

Let $\mathcal{U}_{\alpha}^{d}$ be such a subset of $\mathcal{U}^{d}$ that it's elements have the operator $H_{\alpha}$ eigenvectors $\psi_{\alpha}^{(j)}$ in the capacity of the channel $\alpha$ components: $\mathcal{U}_{\alpha}^{d}=\left\{U_{j}: U_{j}=\left\{u_{1}^{(j)}, u_{2}^{(j)}\right\}, u_{\alpha}^{(j)}=\psi_{\alpha}^{(j)}\right\}$. By Theorem 2, we have $\mathcal{U}_{1}^{d} \cup \mathcal{U}_{2}^{d}=\mathcal{U}^{d}$.

Theorem 3 Let $H_{\beta}=A_{\beta}+B_{\beta \alpha} Q_{\alpha \beta}$, correspond (for $\left\|B_{\beta \alpha}\right\|_{2}<+\infty$ ) to the same solution $Q_{\alpha \beta}=-Q_{\beta \alpha}^{*}$ of Eqs. (14) and (24) as $H_{\alpha}=A_{\alpha}+B_{\alpha \beta} Q_{\beta \alpha}$, and the conditions (23) are valid. Let $z_{j} \in \sigma_{d}\left(H_{\alpha}\right)$ and $H_{\alpha} u_{\alpha}^{(j)}=z_{j} u_{\alpha}^{(j)}$ with $u_{\alpha}^{(j)}$, the channel $\alpha$ component of the eigenvector $U_{j}=\left\{u_{\alpha}^{(j)}, u_{\beta}^{(j)}\right\}$ of the operator $\mathbf{H}, \mathbf{H} U_{j}=z_{j} U_{j}$. Then either $z_{j} \notin \sigma_{d}\left(H_{\beta}\right), \beta \neq \alpha$, or (if $\left.z_{j} \in \sigma_{d}\left(H_{\beta}\right)\right)$ the vector $u_{\beta}^{(j)}$ is not an eigenvector of $H_{\beta}$.

Corollary $4 \mathcal{U}_{1}^{d} \cap \mathcal{U}_{2}^{d}=\emptyset$. 
Statement of Theorem 3 means that discrete spectrum $\sigma_{d}(\mathbf{H})$ is distributed between discrete spectra $\sigma_{d}\left(H_{1}\right)$ and $\sigma_{d}\left(H_{2}\right)$ in such a way that operators $H_{1}$ and $H_{2}$ have not "common" eigenvectors $U_{j}=\left\{u_{1}^{(j)}, u_{2}^{(j)}\right\}$ : simultaneously, component $u_{1}^{(j)}$ can not be eigenvector for $H_{1}$, and $u_{2}^{(j)}$ with the same $j$, for $H_{2}$.

Proof of the Theorem will be given by contradiction.

Let us suppose that $\psi_{\alpha}^{(j)}=u_{\alpha}^{(j)}$ is an eigenvector of $H_{\alpha}$ corresponding to $z_{j}$ i.e.

$$
\left(A_{\alpha}+B_{\alpha \beta} Q_{\beta \alpha}-z_{j}\right) \psi_{\alpha}^{(j)}=0 .
$$

If $z_{j} \in \sigma_{\alpha}=\sigma\left(A_{\alpha}\right)$ then automatically $z_{j} \notin \sigma_{d}\left(H_{\beta}\right)$ since due to conditions (23) we have $\sigma\left(H_{\beta}\right) \cap \sigma\left(A_{\alpha}\right)=\emptyset$. Thus in the case where $z_{j} \in \sigma_{\alpha}$ the assertion of Theorem is valid.

Let $z_{j} \notin \sigma\left(A_{\alpha}\right)$. In this case we can rewrite Eq. (30) in the form

$$
\psi_{\alpha}^{(j)}=-\left(A_{\alpha}-z_{j}\right)^{-1} B_{\alpha \beta} Q_{\beta \alpha} \psi_{\alpha}^{(j)} .
$$

Let $y_{\beta}^{(j)}=Q_{\beta \alpha} \psi_{\alpha}^{(j)}$. It follows from (31) that

$$
y_{\beta}^{(j)}+Q_{\beta \alpha}\left(A_{\alpha}-z_{j}\right)^{-1} y_{\beta}^{(j)}=0 .
$$

We will show that the vector $y_{\beta}^{(j)}$ is a solution of the initial spectral problem (2) in the channel $\beta$ at $z=z_{j}$ and $\tilde{U}_{j}=\left\{\psi_{\alpha}^{(j)}, y_{\beta}^{(j)}\right\}$ is an eigenvector of $\mathbf{H}, \mathbf{H} \tilde{U}_{j}=z_{j} \tilde{U}_{j}$. To do this, we act on both parts of Eq. (32) by $H_{\beta}^{*}-z_{j}$ remembering that, according to (25), $Q_{\beta \alpha}=-Q_{\alpha \beta}^{*}$ $=-\int_{\sigma_{\alpha}}\left(H_{\beta}^{*}-\lambda\right)^{-1} B_{\beta \alpha} E_{\alpha}(d \lambda)$. We obtain

$$
\left(H_{\beta}^{*}-z_{j}\right) y_{\beta}^{(j)}+\int_{\sigma_{\alpha}}\left(H_{\beta}^{*}-z_{j}\right)\left(H_{\beta}^{*}-\lambda\right)^{-1}\left(z_{j}-\lambda\right)^{-1} B_{\beta \alpha} E_{\alpha}(d \lambda) B_{\alpha \beta} y_{\beta}^{(j)}=0 .
$$

Using the identity $(H-z)(H-\lambda)^{-1}(z-\lambda)^{-1}=(z-\lambda)^{-1}-(H-\lambda)^{-1}$ we find

$$
\left(H_{\beta}^{*}-z_{j}\right) y_{\beta}^{(j)}+\int_{\sigma_{\alpha}}\left[\left(z_{j}-\lambda\right)^{-1}-\left(H_{\beta}^{*}-z_{j}\right)\right] B_{\beta \alpha} E_{\alpha}(d \lambda) B_{\alpha \beta} y_{\beta}^{(j)}=0
$$

or, and it is the same,

$$
\left(H_{\beta}^{*}-z_{j}\right) y_{\beta}^{(j)}-B_{\alpha \beta}\left(A_{\alpha}-z_{j}\right)^{-1} B_{\alpha \beta} y_{\beta}^{(j)}+Q_{\beta \alpha} B_{\alpha \beta} y_{\beta}^{(j)}=0
$$

However $H_{\beta}^{*}=A_{\beta}-Q_{\beta \alpha} B_{\alpha \beta}$. Hence the relation (33) turns in equation (2) for the channel $\beta$,

$$
\left[A_{\beta}-B_{\beta \alpha}\left(A_{\alpha}-z_{j}\right)^{-1} B_{\alpha \beta}-z_{j}\right] y_{\beta}^{(j)}=0 .
$$

So, we have proved that $y_{\beta}^{(j)}$ is a solution of the initial problem in the channel $\beta$ and we did deal with an eigenvector $U_{j}=\left\{u_{\alpha}^{(j)}, u_{\beta}^{(j)}\right\}$ of the operator $\mathbf{H}$ having the components $u_{\alpha}^{(j)}=\psi_{\alpha}^{(j)}$ and $u_{\beta}^{(j)}=y_{\alpha}^{(j)}$.

Let us show that $y_{\beta}^{(j)}$ can not be an eigenvector of $H_{\beta}$ corresponding to the eigenvalue $z_{j}$. Actually, due to (32) we have

$$
\mathrm{a} \equiv\left\langle y_{\beta}^{(j)}+Q_{\beta \alpha}\left(A_{\alpha}-z_{j}\right)^{-1} B_{\alpha \beta} y_{\beta}^{(j)}, y_{\beta}^{(j)}\right\rangle=0 .
$$


On the other hand

$$
\mathrm{a}=\left\|y_{\beta}^{(j)}\right\|^{2}+\left\langle\left(A_{\alpha}-z_{j}\right)^{-1} B_{\alpha \beta} y_{\beta}^{(j)}, Q_{\beta \alpha}^{*} y_{\beta}^{(j)}\right\rangle .
$$

If $y_{\beta}^{(j)}$ is an eigenvector of $H_{\beta}, H_{\beta} y_{\beta}^{(j)}=z_{j} y_{\beta}^{(j)}$, then

$$
Q_{\beta \alpha}^{*} y_{\beta}^{(j)}=-Q_{\alpha \beta} y_{\beta}^{(j)}=-\int_{\sigma_{\alpha}} E_{\alpha}(d \lambda) B_{\alpha \beta}\left(H_{\beta}-\lambda\right)^{-1} y_{\beta}^{(j)}=\left(A_{\alpha}-z_{j}\right)^{-1} B_{\alpha \beta} y_{\beta}^{(j)}
$$

It means that

$$
\mathrm{a}=\left\|y_{\beta}^{(j)}\right\|^{2}+\left\|\left(A_{\alpha}-z_{j}\right)^{-1} B_{\alpha \beta} y_{\beta}^{(j)}\right\|^{2} \geq\left\|y_{\beta}^{(j)}\right\|^{2}
$$

Since $\mathrm{a}=0$ we get $y_{\beta}^{(j)}=0$ and, due to $(31), \psi_{\alpha}^{(j)}=0$. However, by supposition, $\psi_{\alpha}^{(j)} \neq 0$. Thus, we come to a contradiction and $y_{\beta}^{(j)}$ can not be an eigenvector of $H_{\beta}$. And so, if $z_{j} \in \sigma_{d}\left(H_{\alpha}\right)$ and $H_{\alpha} u_{\alpha}^{(j)}=z_{j} u_{\alpha}^{(j)}$ then $u_{\beta}^{(j)}$ is not an eigenvector of $H_{\beta}$. The proof of Theorem 3 is completed.

Let us pay attention to the continuous spectrum of $H_{\alpha}$ assuming here that $B_{\alpha \beta} \in \mathcal{B}_{\theta \gamma}^{\beta \alpha}$, $\theta>\frac{1}{2}, \gamma>\frac{1}{2}$, and consequently, $Q_{\alpha \beta} \in \mathcal{B}_{\theta \gamma}^{\beta \alpha}, \alpha, \beta=1,2, \beta \neq \alpha$.

Consider at $\lambda^{\prime} \in \sigma_{\alpha}^{c}$ the integral equations

$$
\psi_{\alpha}^{( \pm)}\left(\lambda, \lambda^{\prime}\right)=I_{\alpha}^{c} \delta\left(\lambda-\lambda^{\prime}\right)-\left[\left(A_{\alpha}-\lambda^{\prime} \mp i 0\right)^{-1} W_{\alpha} \psi_{\alpha}^{( \pm)}\right]\left(\lambda, \lambda^{\prime}\right), \quad \alpha=1,2,
$$

where as usually $W_{\alpha}=B_{\alpha \beta} Q_{\beta \alpha}$. Since $W_{\alpha} \in \mathcal{B}_{\theta \gamma}^{\alpha \alpha}$, the integral operator with the kernel $\frac{W_{\alpha}\left(\lambda, \lambda^{\prime}\right)}{\lambda-\lambda^{\prime} \mp i 0}$ is compact in $\mathcal{M}_{\theta^{\prime} \gamma^{\prime}}, \frac{1}{2}<\theta^{\prime}<\theta, 0<\gamma^{\prime}<\gamma$ (cf. Refs. [20], 21]). If $\lambda^{\prime} \notin \sigma_{d}\left(H_{\alpha}\right)$ then Eq. (34) for $\psi_{\alpha}^{(+)}$as well as for $\psi_{\alpha}^{(-)}$is uniquely solvable (see Ref. [21]) in the class of the form (10) distributions.

Denote by $\Psi_{\alpha}^{( \pm)}, \Psi_{\alpha}^{( \pm)}: \mathcal{H}_{\alpha}^{c} \rightarrow \mathcal{H}_{\alpha}$, the integral operator with the kernel $\psi_{\alpha}^{( \pm)}\left(\lambda, \lambda^{\prime}\right)$. The operator $\Psi_{\alpha}^{( \pm)}$is bounded and $\Psi_{\alpha}^{( \pm)} \mathcal{D}\left(A_{\alpha}^{(0)}\right) \subseteq \mathcal{D}\left(H_{\alpha}\right)$ [20], [21]. It follows from (34) that $\Psi_{\alpha}^{( \pm)}$has the property $H_{\alpha} \Psi_{\alpha}^{( \pm)}=\Psi_{\alpha}^{( \pm)} A_{\alpha}^{(0)}$. Thus, $Q_{\beta \alpha} \Psi_{\alpha}^{( \pm)}\left(\cdot, \lambda^{\prime}\right)=\left(\lambda^{\prime}-A_{\beta}\right)^{-1} B_{\beta \alpha} \Psi_{\alpha}^{( \pm)}\left(\cdot, \lambda^{\prime}\right)$. Substitution of this expression in (34) shows that $\psi_{\alpha}^{( \pm)}$satisfies (9). Due to the uniqueness of Eq. (9) solution at $\lambda^{\prime} \notin \sigma_{d}(\mathbf{H})$ we have $\psi_{\alpha}^{( \pm)}\left(\lambda, \lambda^{\prime}\right)=u_{\alpha \alpha}^{( \pm)}\left(\lambda, \lambda^{\prime}\right)$. This means that each eigenfunction $u_{\alpha \alpha}^{( \pm)}\left(\lambda, \lambda^{\prime}\right), \lambda^{\prime} \in \sigma_{\alpha}^{c}, \lambda^{\prime} \notin \sigma_{d}(\mathbf{H})$ of the initial spectral problem problem (2) is also an eigenfunction of $H_{\alpha}$.

Consider the functions $\tilde{\psi}_{\alpha}^{(j)}=\psi_{\alpha}^{(j)}-Q_{\alpha \beta} u_{\beta}^{(j)}$ and $\tilde{\psi}_{\alpha}^{( \pm)}\left(\cdot, \lambda^{\prime}\right)=\psi_{\alpha}^{( \pm)}\left(\cdot, \lambda^{\prime}\right)-Q_{\alpha \beta} u_{\beta \alpha}^{( \pm)}\left(\cdot, \lambda^{\prime}\right), \lambda^{\prime} \in \sigma_{\alpha}^{c}$. Let $\tilde{\Psi}_{\alpha}^{( \pm)}, \tilde{\Psi}_{\alpha}^{( \pm)}: \mathcal{H}_{\alpha}^{c} \rightarrow \mathcal{H}_{\alpha}$, be the integral operator with the kernel $\tilde{\psi}_{\alpha}^{( \pm)}\left(\lambda, \lambda^{\prime}\right)$.

Theorem 4 The functions $\tilde{\psi}_{\alpha}^{(j)}$ (with $j$ such that $U_{j} \in \mathcal{U}_{\alpha}^{d}$ ) are eigenfunctions of adjoint operator $H_{\alpha}^{*}, H_{\alpha}^{*}=A_{\alpha}+Q_{\beta \alpha}^{*} B_{\beta \alpha}$, discrete spectrum, $H_{\alpha}^{*} \tilde{\psi}_{\alpha}^{(j)}=z_{j} \tilde{\psi}_{\alpha}^{(j)}$. Operators $\tilde{\Psi}_{\alpha}^{( \pm)}$have the property $H_{\alpha}^{*} \tilde{\Psi}_{\alpha}^{( \pm)}=\tilde{\Psi}_{\alpha}^{( \pm)} A_{\alpha}^{(0)}$. At the same time the orthogonality relations take place: $\left\langle\psi_{\alpha}^{(j)}, \tilde{\psi}_{\alpha}^{(k)}\right\rangle=\delta_{j k}, \Psi_{\alpha}^{( \pm) *} \tilde{\Psi}_{\alpha}^{( \pm)}=\left.I_{\alpha}\right|_{\mathcal{H}_{\alpha}^{c}}, \tilde{\Psi}_{\alpha}^{( \pm) *} \psi_{\alpha}^{(j)}=0$ and $\Psi_{\alpha}^{( \pm) *} \tilde{\psi}_{\alpha}^{(j)}=0$. Also, the following completeness relations are valid,

$$
\sum_{j: U_{j} \in \mathcal{U}_{\alpha}^{d}} \psi_{\alpha}^{(j)}\left\langle\cdot, \tilde{\psi}_{\alpha}^{(j)}\right\rangle+\Psi_{\alpha}^{( \pm)} \tilde{\Psi}_{\alpha}^{( \pm) *}=I_{\alpha}, \quad \alpha=1,2
$$


Proof. Show for example that

$$
H_{\alpha}^{*} \tilde{\psi}_{\alpha}^{(j)}=z_{j} \tilde{\psi}_{\alpha}^{(j)}
$$

(remember that $z_{j} \in \mathbf{R}$ ). We have

$$
\begin{gathered}
H_{\alpha}^{*} \tilde{\psi}_{\alpha}^{(j)}=\left(A_{\alpha}-Q_{\alpha \beta} B_{\beta \alpha}\right)\left(\tilde{\psi}_{\alpha}^{(j)}-Q_{\alpha \beta} u_{\beta}^{(j)}\right)= \\
=\left(A_{\alpha}-Q_{\alpha \beta} B_{\beta \alpha}\right) \psi_{\alpha}^{(j)}-\left(A_{\alpha} Q_{\alpha \beta}-Q_{\alpha \beta} B_{\beta \alpha} Q_{\alpha \beta}\right) u_{\beta}^{(j)} .
\end{gathered}
$$

Note that $A_{\alpha}=H_{\alpha}-B_{\alpha \beta} Q_{\beta \alpha}$ and, hence,

$$
\left(A_{\alpha}-Q_{\alpha \beta} B_{\beta \alpha}\right) \psi_{\alpha}^{(j)}=z_{j} \psi_{\alpha}^{(j)}-\left(B_{\alpha \beta} Q_{\beta \alpha}+Q_{\alpha \beta} B_{\beta \alpha}\right) \psi_{\alpha}^{(j)} .
$$

The second term in the right part of (38) may be easily expressed by $u_{\beta}^{(j)}$. Actually, $u_{\beta}^{(j)}=$ $-\left(A_{\beta}-z_{j}\right)^{-1} B_{\beta \alpha} \psi_{\alpha}^{(j)}$ [here, we use again the property $\sigma\left(H_{\alpha}\right) \cap \sigma_{\beta}=\emptyset$ following from (23))]. Since Eqs. (13) and $H_{\alpha} \psi_{\alpha}^{(j)}=z_{j} \psi_{\alpha}^{(j)}$ take place, we find $Q_{\beta \alpha} \psi_{\alpha}^{(j)}=B_{\alpha \beta} u_{\beta}^{(j)}$. Thus,

$$
\begin{aligned}
\left(B_{\alpha \beta} Q_{\beta \alpha}+Q_{\alpha \beta} B_{\beta \alpha}\right) \psi_{\alpha}^{(j)} & =B_{\beta \alpha} Q_{\beta \alpha} \psi_{\alpha}^{(j)}+Q_{\alpha \beta}\left(A_{\beta}-z_{j}\right)\left(A_{\beta}-z_{j}\right)^{-1} B_{\beta \alpha} \psi_{\alpha}^{(j)}= \\
& =B_{\beta \alpha} u_{\beta}^{(j)}-Q_{\alpha \beta}\left(A_{\beta}-z_{j}\right) u_{\beta}^{(j)} .
\end{aligned}
$$

Substituting the expressions obtained into (38) and then into (37), we get

$$
H_{\alpha}^{*} \tilde{\psi}_{\alpha}^{(j)}=z_{j}\left(\psi_{\alpha}^{(j)}-Q_{\alpha \beta} u_{\beta}^{(j)}\right)+\left[-B_{\alpha \beta}+Q_{\alpha \beta} A_{\beta}-A_{\alpha} Q_{\alpha \beta}+Q_{\alpha \beta} B_{\beta \alpha} Q_{\alpha \beta}\right] u_{\beta}^{(j)} .
$$

According to the equations (24), the expression in the square brackets is equal to zero and we come to (36).

The equalities $H_{\alpha}^{*} \tilde{\psi}_{\alpha}^{( \pm)}\left(\cdot, \lambda^{\prime}\right)=\lambda^{\prime} \tilde{\psi}_{\alpha}^{( \pm)}\left(\cdot, \lambda^{\prime}\right), \lambda^{\prime} \in \sigma_{\alpha}^{c}$, are proved quite analogously.

The orthogonality relations $\left\langle\psi_{\alpha}^{(j)}, \tilde{\psi}_{\alpha}^{(k)}\right\rangle=\delta_{j k}, \tilde{\Psi}_{\alpha}^{( \pm) *} \psi_{\alpha}^{(j)}=0$ and $\Psi_{\alpha}^{( \pm) *} \tilde{\psi}_{\alpha}^{(j)}=0$ are trivial. Proofs of the relation $\Psi_{\alpha}^{( \pm) *} \tilde{\Psi}_{\alpha}^{( \pm)}=\left.I_{\alpha}\right|_{\mathcal{H}_{\alpha}^{c}}$, and the equality (35) are very similar. Both these proofs are based on use of properties of the wave operators $U^{( \pm)}$. As a sample, we give a proof of the completeness relation (35).

Consider the operator

$$
\begin{gathered}
\mathcal{A}=\sum_{j: U_{j} \in \mathcal{U}_{\alpha}^{d}} \psi_{\alpha}^{(j)}\left\langle\cdot, \tilde{\psi}_{\alpha}^{(j)}\right\rangle+\Psi_{\alpha} \tilde{\Psi}_{\alpha}^{*}= \\
=\sum_{j: U_{j} \in \mathcal{U}_{\alpha}^{d}} \psi_{\alpha}^{(j)}\left\langle\cdot, \psi_{\alpha}^{(j)}-Q_{\alpha \beta} u_{\beta}^{(j)}\right\rangle+\Psi_{\alpha}\left[\Psi_{\alpha}^{*}-\left(Q_{\alpha \beta} u_{\beta \alpha}\right)^{*}\right] .
\end{gathered}
$$

For convenience, we omit signs " \pm " in notations of $\Psi_{\alpha}^{( \pm)} \equiv u_{\alpha \alpha}^{( \pm)}, u_{\beta \alpha}^{( \pm)}$and $\tilde{\Psi}_{\alpha}^{( \pm)}$taking in mind for example the case of sign "+". We have from (39):

$$
\mathcal{A}=\sum_{j: U_{j} \in \mathcal{U}_{\alpha}^{d}} \psi_{\alpha}^{(j)}\left\langle\cdot, \psi_{\alpha}^{(j)}\right\rangle+\Psi_{\alpha} \Psi_{\alpha}^{*}-\sum_{j: U_{j} \in \mathcal{U}_{\alpha}^{d}} \psi_{\alpha}^{(j)}\left\langle\cdot, Q_{\alpha \beta} u_{\beta}^{(j)}\right\rangle-\Psi_{\alpha} u_{\beta \alpha}^{*} Q_{\alpha \beta}^{*} .
$$

It follows from the completeness relations $U^{( \pm) *} U^{( \pm)}=I-P$ for wave operators $U^{( \pm)}$that 


$$
\Psi_{\alpha} u_{\beta \alpha}^{*} \equiv u_{\alpha \alpha} u_{\beta \alpha}^{*}=-u_{\alpha \beta} u_{\beta \beta}^{*}-\sum_{z_{j} \in \sigma(\mathbf{H})} u_{\alpha}^{(j)}\left\langle\cdot, u_{\beta}^{(j)}\right\rangle
$$

Since $u_{\beta \beta}^{*} Q_{\alpha \beta}^{*}=\left(Q_{\alpha \beta} u_{\beta \beta}\right)^{*}=u_{\alpha \beta}^{*}$, we can write with a help of (40) that

$$
\mathcal{A}=u_{\alpha \alpha} u_{\alpha \alpha}^{*}+u_{\alpha \beta} u_{\alpha \beta}^{*}+\sum_{j: U_{j} \in \mathcal{U}_{\alpha}^{d}} \psi_{\alpha}^{(j)}\left\langle\cdot, \psi_{\alpha}^{(j)}\right\rangle+\sum_{\substack{z_{j} \in \sigma_{d}(\mathbf{H}) \\ U_{j} \notin \mathcal{U}_{\alpha}^{d}}} u_{\alpha}^{(j)}\left\langle\cdot, Q_{\alpha \beta} u_{\beta}^{(j)}\right\rangle .
$$

In the last sum, the conditions $z_{j} \in \sigma_{d}(\mathbf{H})$ and $U_{j} \notin \mathcal{U}_{\alpha}^{d}$ mean really that we deal with any $j$ such that $U_{j} \in \mathcal{U}_{\beta}^{d}$. This follows from the equalities $\mathcal{U}_{1}^{d} \cup \mathcal{U}_{2}^{d}=\mathcal{U}^{d}$ and $\mathcal{U}_{1}^{d} \cap \mathcal{U}_{2}^{d}=\emptyset$ (see Theorem 3 and Corollary $⿴)$. For $U_{j} \in \mathcal{U}_{\beta}^{d}$, the vector $u_{\beta}^{(j)}$ is eigenfunction of $H_{\beta}, u_{\beta}^{(j)}=\psi_{\beta}^{(j)}$, and $Q_{\alpha \beta} u_{\beta}^{(j)}=Q_{\alpha \beta} \psi_{\beta}^{(j)}=u_{\alpha}^{(j)}$. Thus, $\mathcal{A}$ turns in

$$
\mathcal{A}=u_{\alpha \alpha} u_{\alpha \alpha}^{*}+u_{\alpha \beta} u_{\beta \alpha}^{*}+\sum_{z_{j} \in \sigma_{d}(\mathbf{H})} u_{\alpha}^{(j)}\left\langle\cdot, u_{\alpha}^{(j)}\right\rangle=\left(U^{( \pm)} U^{( \pm) *}+P\right)_{\alpha \alpha} .
$$

Since $U^{( \pm)} U^{( \pm) *}+P=I$ we find $\mathcal{A}=I_{\alpha}$ and this completes the proof of Theorem $甘$.

Theorem 1 means in particular that part $H_{\alpha}^{c}$ of operator $H_{\alpha}$ acting in the invariant subspace corresponding to it's continuous spectrum $\sigma_{\alpha}^{c}$, is similar to the operator $A_{\alpha}^{(0)}, H_{\alpha}^{c}=$ $\Psi_{\alpha}^{( \pm)} A_{\alpha}^{(0)} \tilde{\Psi}_{\alpha}^{( \pm) *}$, and spectrum $\sigma_{\alpha}^{c}$ is absolutely continuous.

\section{INNER PRODUCT MAKING NEW HAMILTONIANS SELF-ADJOINT}

We introduce now a new inner product $[., .]_{\alpha}$ in $\mathcal{H}_{\alpha},\left[f_{\alpha}, g_{\alpha}\right]_{\alpha}=\left\langle X_{\alpha} f_{\alpha}, g_{\alpha}\right\rangle, f_{\alpha}, g_{\alpha} \in \mathcal{H}_{\alpha}$, with $X_{\alpha}$ defined as in Theorem 2, $X_{\alpha}=I_{\alpha}+Q_{\alpha \beta} Q_{\alpha \beta}^{*}, \alpha=1,2$. The operator $X_{\alpha}$ is positive definite, $X_{\alpha} \geq I_{\alpha}$. This means that $[., .]_{\alpha}$ satisfies all the axioms of inner product.

Theorem 5 The operator $H_{\alpha}, \alpha=1,2$, is self-adjoint on $\mathcal{D}\left(A_{\alpha}\right)$ in respect with the inner product $[., .]_{\alpha}$.

Proof. It follows from Theorem 2 that operator $\mathbf{H}^{\prime}$ is self-adjoint in $\mathcal{H}=\mathcal{H}_{1} \oplus \mathcal{H}_{2}$ in respect with the inner product $[.,],.[f, g]=[X f, g]$ with $X=\operatorname{diag}\left\{X_{1}, X_{2}\right\}$. Did, since $\mathcal{Q}^{-1}=\mathcal{Q}^{*} X^{-1}=X^{-1} \mathcal{Q}^{*}$, we have for $f, g \in \mathcal{D}\left(\mathbf{H}^{\prime}\right)=\mathcal{D}(\mathbf{H})=\mathcal{D}\left(A_{1}\right) \oplus \mathcal{D}\left(A_{2}\right)$ :

$$
\begin{gathered}
{\left[\mathbf{H}^{\prime} f, g\right]=\left\langle X \mathcal{Q}^{-1} \mathbf{H} \mathcal{Q} f, g\right\rangle=\left\langle X \cdot X^{-1} \mathcal{Q}^{*} \mathbf{H} \mathcal{Q} f, g\right\rangle=} \\
=\left\langle f, \mathcal{Q}^{*} \mathbf{H} \mathcal{Q} g\right\rangle=\left\langle f, X \cdot X^{-1} \mathcal{Q}^{*} \mathbf{H} \mathcal{Q} g\right\rangle=\left[f, \mathbf{H}^{\prime} g\right] .
\end{gathered}
$$

Here, we used the fact that in the case of (23), $\mathcal{Q} f \in \mathcal{D}\left(A_{1}\right) \oplus \mathcal{D}\left(A_{2}\right)$ if $f \in \mathcal{D}\left(A_{1}\right) \oplus \mathcal{D}\left(A_{2}\right)$.

Taking elements $f, g$ in the equality $\left[\mathbf{H}^{\prime} f, g\right]=\left[f, \mathbf{H}^{\prime} g\right]$ in the form $f=\left\{f_{1}, 0\right\}, g=\left\{g_{1}, 0\right\}$ or $f=\left\{0, f_{2}\right\}, g=\left\{0, g_{2}\right\}$ with one of the components equal to zero and $f_{\alpha}, g_{\alpha} \in \mathcal{D}\left(A_{\alpha}\right)$, $\alpha=1,2$, one comes to the statement of Theorem.

Remark. This Theorem may be proved also in another way making use of the equality

$$
I_{\alpha}+Q_{\alpha \beta} Q_{\alpha \beta}^{*}=\sum_{j: U_{j} \in \mathcal{U}_{\alpha}^{d}} \tilde{\psi}_{\alpha}^{(j)}\left\langle\cdot, \tilde{\psi}_{\alpha}^{(j)}\right\rangle+\tilde{\Psi}_{\alpha}^{( \pm)} \tilde{\Psi}_{\alpha}^{( \pm) *}
$$


which is valid for both signs "+" and "-". In this case, a self-adjointness of $H_{\alpha}$ in respect with $[\cdot, \cdot]_{\alpha}$ follows from the fact that it's spectrum is real and also from relations $H_{\alpha}^{*} \tilde{\Psi}_{\alpha}^{( \pm)} \tilde{\Psi}_{\alpha}^{( \pm) *}=\tilde{\Psi}_{\alpha}^{( \pm)} A_{\alpha}^{(0)} \tilde{\Psi}_{\alpha}^{( \pm) *}=\tilde{\Psi}_{\alpha}^{( \pm)} \tilde{\Psi}_{\alpha}^{( \pm) *} H_{\alpha}$. The equality (41) itself is proved by calculating it's right part in the same way as it was done where the completeness relations (35) were established (see proof of Theorem 1 ).

\section{SCATTERING PROBLEM}

We establish now that operators $\Psi_{\alpha}^{(+)}$and $\Psi_{\alpha}^{(-)}$play the same important role describing a time asymptotics of solutions of the Schrödinger equation

$$
i \frac{d}{d t} f_{\alpha}(t)=H_{\alpha} f_{\alpha}(t)
$$

as in the usual self-adjoint case [20], 21].

Theorem 6 Operator $U_{\alpha}(t)=\exp \left(i H_{\alpha} t\right) \exp \left(-i A_{\alpha}^{(0)} t\right)$ converges strongly if $t \rightarrow \mp \infty$, in respect with the norm $\|\cdot\|_{\alpha}^{X}$ corresponding to the inner product $[\cdot, \cdot]_{\alpha}$ in $\mathcal{H}_{\alpha}$. The limit is equal to $s-\lim _{t \rightarrow \mp \infty} U_{\alpha}(t)=\Psi_{\alpha}^{( \pm)}$.

Since the norms $\|\cdot\|_{\alpha}^{X}$ and $\|\cdot\|$ in $\mathcal{H}_{\alpha}$ are equivalent, $\|f\| \leq\|f\|_{\alpha}^{X} \leq\left(1+\left\|Q_{\alpha \beta}\right\|\right.$. $\left.\left\|Q_{\beta \alpha}\right\|\right)^{1 / 2}\|f\|$, the same statement takes place also in respect with the initial norm $\|\cdot\|$.

Theorem 7 For any element $f_{\alpha}^{(-)} \in \mathcal{H}_{\alpha}^{c}$ one can find such unique element $f_{\alpha}^{(0)}$ that solution $f_{\alpha}(t)=\exp \left(-i H_{\alpha} t\right) f_{\alpha}^{(0)}$ of Eq. (壮) satisfies the asymptotic condition

$$
\lim _{t \rightarrow-\infty}\left\|f_{\alpha}(t)-\exp \left(-i A_{\alpha}^{(0)} t\right) f_{\alpha}^{(-)}\right\|_{\alpha}^{X}=0 .
$$

There exists the unique element $f_{\alpha}^{(+)} \in \mathcal{H}_{\alpha}^{c}$ such that

$$
\lim _{t \rightarrow+\infty}\left\|f_{\alpha}(t)-\exp \left(-i A_{\alpha}^{(0)} t\right) f_{\alpha}^{(+)}\right\|_{\alpha}^{X}=0 .
$$

Elements $f_{\alpha}^{(-)}$and $f_{\alpha}^{(+)}$are connected by the relation $f_{\alpha}^{(+)}=S^{(\alpha)} f_{\alpha}^{(-)}$with $S^{(\alpha)}=\Psi_{\alpha}^{(-)-1} \Psi_{\alpha}^{(+)}=\tilde{\Psi}_{\alpha}^{(-) *} \Psi_{\alpha}^{(+)}=\Psi_{\alpha}^{(-) *} X_{\alpha} \Psi_{\alpha}^{(+)}$.

We do not give here proofs of the Theorems 6 and 7 because they are exactly the same as in the case of one-particle Schrödinger operator in Ref. [29]

Theorem 7 gives the non-stationary formulation of the scattering problem for a system described by Hamiltonian $H_{\alpha}$. Moreover $S^{(\alpha)}$ is a scattering operator for this system.

Theorem 8 Scattering operator $S^{(\alpha)}$ coincides with the component $s_{\alpha \alpha}$ of the scattering operator $S, S=U^{(-) *} U^{(+)}$, for a system described by the two-channel Hamiltonian $\mathbf{H}$.

Proof. Let us show that operator $S^{(\alpha)}$ has the kernel $s_{\alpha \alpha}\left(\lambda, \lambda^{\prime}\right)$ given by Eq. (11). To do this, remember that $\tilde{\Psi}_{\alpha}^{(-)}=\Psi_{\alpha}^{(-)}-Q_{\alpha \beta} u_{\beta \alpha}^{(-)}$(see Theorem 四). Therefore,

$$
S^{(\alpha)}=\left(\Psi_{\alpha}^{(-) *}-u_{\beta \alpha}^{(-) *} Q_{\alpha \beta}^{*}\right) \Psi_{\alpha}^{(+)}=\Psi_{\alpha}^{(-) *} \Psi_{\alpha}^{(+)}+u_{\beta \alpha}^{(-) *} Q_{\beta \alpha} \Psi_{\alpha}^{(+)}=u_{\alpha \alpha}^{(-) *} u_{\alpha \alpha}^{(+)}+u_{\beta \alpha}^{(-) *} u_{\beta \alpha}^{(+)} .
$$


Here, we have used the properties $\Psi_{\alpha}^{( \pm)}=u_{\alpha \alpha}^{( \pm)}, Q_{\alpha \beta}^{*}=-Q_{\beta \alpha}$ and $Q_{\beta \alpha} \Psi_{\alpha}^{(+)}=u_{\beta \alpha}^{(+)}$established above. Since

$$
u_{\alpha \alpha}^{(-) *} u_{\alpha \alpha}^{(+)}+u_{\beta \alpha}^{(-) *} u_{\beta \alpha}^{(+)}=\left(U^{(-) *} U^{(+)}\right)_{\alpha \alpha}=s_{\alpha \alpha},
$$

we come to the statement of Theorem. The proof of Theorem 7 is completed.

A kernel of the scattering operator $S^{(\alpha)}$ may be presented also in a usual way (11) in terms of the $t$-matrix $t_{\alpha}(z)=W_{\alpha}-W_{\alpha}\left(H_{\alpha}-z\right)^{-1} W_{\alpha}$, taken on the energy-shell. Note that $t_{\alpha}(z)$ differs from $T_{\alpha \alpha}(z)$ introduced in Sec. [1]. Did, easy calculations show that

$$
t_{\alpha}(z)=B_{\alpha \beta}\left[I_{\beta}+Q_{\beta \alpha}\left(A_{\alpha}-z\right)^{-1} B_{\alpha \beta}\right]^{-1} Q_{\beta \alpha} .
$$

Using the basic equation (24) one can rewrite (43) in the form

$$
t_{\alpha}(z)=T_{\alpha \alpha}(z)+\tilde{t}_{\alpha}(z)
$$

where

$$
\tilde{t}_{\alpha}(z)=B_{\alpha \beta}\left[A_{\beta}-B_{\beta \alpha}\left(A_{\alpha}-z\right)^{-1} B_{\alpha \beta}\right]^{-1} Q_{\alpha \beta}\left(A_{\alpha}-z\right) \not \equiv 0 .
$$

However the additional term $\tilde{t}_{\alpha}(z)$ is evidently disappearing on the energy-shell due to presence of the difference $A_{\alpha}-z$ as an end factor. Actually, in the diagonal representation (6), (7), $A_{\alpha}-z$ acts as the factor $\lambda-z$ vanishing at $z=\lambda+i 0$. Therefore, kernels of $t$-matrices $t_{\alpha}$ and $T_{\alpha \alpha}$ coincide on the energy surface.

Note also that in our case $\sigma_{1}^{c} \cap \sigma_{2}^{c}=\emptyset$. Hence we have $s_{\beta \alpha}=0$ and $S^{(\alpha)}=s_{\alpha \alpha}$ is unitary.

\section{ACKNOWLEDGMENTS}

Author is thankful to Prof. B.S.Pavlov and Dr. K.A.Makarov for support and interest to this work. The author is much indebted to Prof. R.A.Minlos and participants of his seminar in Moscow University for discussion of the presented results. Particularly, the author is grateful to Dr. S.A.Stepin for the literary indications.

The work is supported in part by the International Science Foundation (Grants \#RFB000 and \#RFB300) and the RAS Academy of Natural Sciences. 
[1] R.F.Dashen and J.B.Healy, I.J.Muzinich, Ann. Phys. 102, 1 (1976).

[2] R.L.Jaffe and F.E.Low, Phys. Rev. D 19, 2105 (1979).

[3] Yu.A.Simonov, Yad.Fiz. 36, 722 (1982).

[4] B.O.Kerbikov, Yad. Fiz. 65, 379 (1985).

[5] S.Nakaishi-Maeda and E.W.Schmid, Phys. Rev. C 35, 799 (1987).

[6] I.M.Narodetsky, in III All-Union School on Few Body and Quark-Hadronic Problems (Vilnous, 1986, in Russian), Part I, p. 116.

[7] B.H.J.McKellar and C.M.McKay, Aust.J.Phys.36, 607 (1983).

[8] M.Orlowski and Y.E.Kim, Phys. Rev. D 32, 1376 (1985).

[9] M.A.Braun, Teor.Mat.Fiz.72, 394 (1987).

[10] E.W.Schmid, Helvetica Phys.Acta 60, 394 (1987).

[11] B.S.Pavlov, Teor. Mat. Fiz. 59, 345 (1984).

[12] Yu.A.Kuperin, K.A.Makarov, S.P.Merkuriev and A.K.Motovilov, Yad.Fiz. 48, 358 (1988).

[13] Yu.A.Kuperin, K.A.Makarov, S.P.Merkuriev, A.K.Motovilov and B.S.Pavlov, J. Math. Phys. 31, 1681 (1990).

[14] R.A.Minlos, Teor. Mat. Fiz. 92, 255 (1992) [English translation in Theor. Math. Phys.].

[15] A.K.Motovilov, Teor. Mat. Fiz. 97, 163 (1993) [English translation in Theor. Math. Phys.].

[16] K.N.Ilinsky, A.K.Motovilov and V.M.Uzdin, Helv. Phys. Acta 66, 337 (1993); Poverkhnost (Russian J. Surface) 2, 12 (1994).

[17] A.K.Motovilov, J.Math.Phys. 32/12, 3509 (1991).

[18] A.K.Motovilov, in Proceedings of the XIII Warsaw Sympos. on Elementary Particle Physics /Eds.: Z.Ajduk et al. (World Scientific, 1990), pp. 494-499.

[19] A.K.Motovilov, in Invited Talks of the Intern.Workshop "Mathematical Aspects of the Scattering Theory and Applications" (St.Petersburg, 1991), pp. 101-108.

[20] O.A.Ladyzhenskaya and L.D.Faddeev, Doklady AN SSSR 120, 1187 (1958).

[21] L.D.Faddeev, Trudy Mat. In-ta AN SSSR 73, 292 (1964).

[22] V.A.Malyshev and R.A.Minlos, J. Stat. Phys. 21, 231 (1979).

[23] V.A.Malyshev and R.A.Minlos, Comm. Math. Phys. 82/2, 211 (1981).

[24] V.M.Adamjan and H.Langer, "Spectral properties of a class of rational operator-value functions", accepted for publication in J.Operator Theory.

[25] Remember that if $N_{\alpha} \geq 0$ then Eq. (5) gives a general form of $R$-function on $\mathcal{H}_{\alpha}$, i.e. an analytic at $\operatorname{Im} z \neq 0 \mathbf{B}\left(\mathcal{H}_{\alpha}, \mathcal{H}_{\alpha}\right)$-value function with positive imaginary part for $z: \operatorname{Im} z>0$ (see paper [26] and Refs. therein).

[26] S.N.Naboko, Funktsionalnyi analiz i ego prilozhenia 25, 1 (1991).

[27] M.S.Birman and M.Z.Solomiak, "Spectral Theory of Self-Adjoint Operators in Hilbert Space" (Leningrad U.P., Leningrad, 1980, in Russian).

[28] W.T.Reid, "Riccati differential equations (Academic Press", N.Y.-London, 1972).

[29] L.D.Faddeev, Trudy Mat. In-ta AN SSSR 69, 1-125 (1963) [English translation: "Mathematical aspects of the three-body problem in quantum mechanics". Israel Program for Scientific Translations, Jerusalem, 1965]. 